\title{
Orchestrated Informal Care Coordination
}

An internet-of-things approach for a connected network of tools in support of collective care activities for informal caregivers

Kyungmin Youn 


\section{Orchestrated Informal Care Coordination}

An internet-of-things approach for a connected network of tools in support of collective care activities for informal caregivers

Kyungmin Youn (author)

Mark Baskinger (advisor)

John Zimmerman (advisor) 
Thanks so much to my two advisors, Mark Baskinger and John Zimmerman. Thanks to all the volunteers who participated in the interview. Thanks to my classmates who inspired me, and thanks to my family and Grace for their warm encouragement and tireless support. 


\title{
Orchestrated Informal Care Coordination
}

\begin{abstract}
An internet-of-things approach for a connected network of tools in support of collective care activities for informal caregivers
\end{abstract}

\footnotetext{
Abstract

Many family caregivers find themselves experiencing difficulties in coordinating older adults' health care because it requires not only a lot of time but also a wide scope of responsibilities to coordinate care for their loved ones. While they can reduce their individual burden by sharing care tasks with other family members, there are still many challenges to overcome in maintaining the quality of care when they work together. This thesis project explores opportunities for loT technologies to help informal caregivers better coordinate and communicate care with each other for their loved ones. Based on identified design opportunities, this project proposes the concept of CareBot, a smart home platform consisting of interactive tools in support of collective care activities of family caregivers.
} 
Contents

1. Introduction 9

2. Related Work 10

3. Design Process 12

4. Final Prototype 31

5. Discussion 48

6. Conclusion 49

References $\quad 51$

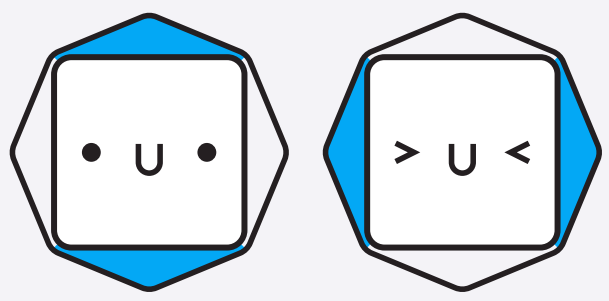





\section{Introduction}

According to the U.S. Census Bureau, there has been a rapid growth in the elderly population in the United States. By 2030, about 1 in 5 U.S. residents are projected to be aged 65 and over. ${ }^{1}$ Already more than 65 million Americans are informal caregivers providing care for an ill, disabled or aged family member. ${ }^{2}$ Due to the continuous growth in the elderly population and the fact that most Americans would rather keep living in their homes than move to care facilities as they get older, ${ }^{3}$ the number of informal caregivers and the importance of in-home care will continually increase.

Often, family caregivers experience difficulties in coordinating older adults' health care because it requires not only a lot of time but also a diverse set of responsibilities to coordinate care for their loved ones. To balance work, life, and caregiver role is an overwhelming task. ${ }^{4}$ While many can reduce their individual burdens by sharing care tasks with other family members or close friends, there are still many challenges to overcome when they work together. As they increase their informal care network, it becomes more difficult for them to stay informed and coordinated. Coordination breakdowns caused by having multiple caregivers who are cooperating to care for the same care recipient result in reduced quality of care.

In the face of this trend, research has been conducted from the design and $\mathrm{HCl}$ communities on how smart home technologies can promote seniors' independent living and safety through the automation of domestic tasks and higher security, and how "Internet of Things" (IOT) technologies can support home health care through monitoring and sensing data of elderly residents. However, despite the fact that family caregivers play a significant role in managing seniors' overall health care, ${ }^{5}$ little has been focused on how family caregivers can benefit from these technologies when it comes to care coordination for their loved ones in their own homes.

This thesis project explores opportunities for loT technologies to help informal caregivers better coordinate and communicate care with each other for their loved ones. To explore this space, this project involved a range of design activities including in-depth interviews with family caregivers, care recipients, and professional care

1 "An Aging Nation: The Older Population in the United States." U.S. Census Bureau, 2012 Population Estimates and 2012 National Projections. May 2014

2 "Caregiving in the U.S. 2015", The National Alliance for Caregiving in collaboration with AARP 3 "Aging in Place." Center for Aging in Place Support. Sep 2010

4 Yunan Chen, Victor Ngo, and Sun Young Park. 2013. Caring for caregivers: designing for integrality. CSCW '13: Proceedings of the 2013 conference on Computer supported cooperative work: 91-102.

5 Giovannetti ER, Wolff J, Xue Q, et al. Difficulty with health care tasks among caregivers of multimorbid older adults. J Gen Intern Med. 2012;27:37-44. 
coordinators, concept generation and needs validation, insight synthesis, and physical form prototyping.

The findings of the qualitative study on informal caregivers and their activities have revealed three main opportunities in which IoT can play a significant role for family caregivers' collective care coordination:

1. facilitating care works by reminding family caregivers of tasks that need to be done and providing instructions on how to accomplish it,

2. having crucial information accessible to everyone in the network of informal caregivers to stay organized and coordinated, and

3. keeping everyone engaged and connected to avoid breakdown caused by poor communication and lack of information.

Finally, based on identified design opportunities, this project suggests the concept of CareBot, a smart home platform consisting of interactive tools in support of collective care activities of family caregivers.

The initial part of this paper presents details of the design process and the findings of the qualitative study, and discuss the opportunities and design implications. In the rest of this paper, it provides an overview of how I designed the concept of CareBot, and how family caregivers can interact with it. In the end, this paper discusses the reflection of this project, limitations, and future work.

\section{Related Work}

\subsection{Home Health Care}

A large amount of $\mathrm{HCl}$ and health informatics research has investigated smart home technologies for senior's independent living (Lee \& Dey 2010; Frisardi et al. 2011; Le et al. 2012; Morris et al. 2013). These studies explored a substantial possibility of smart home technologies for revolutionizing home health care. The studies mainly focused on technological advance for enabling domestic task automation, easier communication, higher security, and better sensing, monitoring, interpreting data of older people at home. It is argued that the emerging technology can empower older adults to address a comprehensive range of medical, health, social, and functional needs (Center for Technology and Aging Report 2014). 


\subsection{Caregiving and Caregivers}

Informal caregivers, such as family and close friends, who take care of their care recipients are particular interest to my project. The definition of an informal caregiver in this project follows the general $\mathrm{HCl}$ health-related literature approach: any nonprofessional person who the patient identified as helping with some aspect of their care (Miller et al. 2015; Kaziunas et al. 2015). They play an important role in older adults' health care (Giovannetti et al. 2012). Especially, the role of informal caregivers is very critical during a patient's hospital stay (Miller et al. 2015). They are very active participants and play diverse roles to coordinate communication with doctors and care for patients' overall wellness needs. Meanwhile, being caregivers can be overwhelmingly demanding (Chen et al. 2013). Caregivers have to constantly balance their personal lives with their caregiver roles, and they usually experience a great magnitude of challenges in their daily lives.

\subsection{Supporting Care Coordination and Communication}

Technologies for parents as informal caregivers have recently received much attention. Lee et al. investigated smart bags for dual-income families to explore the opportunity for technology to help parents to avoid communication breakdowns in parenting (Lee et al. 2007). Liu et al. examined the communication challenges that a patient's caregivers experience when they try to connect with medical service provider after discharge. They designed a mobile application that enables caregivers to share the medical information with other people (Liu et al. 2011). Moncur et al. introduced a system that helps parents better communicate information about their kids to family and friends (Moncur et al. 2014). Suh et al. developed the system that allows parents to keep track of their child's developmental milestones using Twitter (Suh et al. 2014). 


\section{Design Process}

Using a human-centered design approach, I explored various facets that make up the overall experiences of family caregivers who are involved in in-home care coordination, such as their needs, desires, and breakdowns.

My design process included:

- Reviewing academic research from the design and $\mathrm{HCl}$ communities on a range of related works.

- Investigating current tools and services facilitating home health care and informal care coordination.

- Conducting in-depth interviews with 16 people including family caregivers, care recipients, and professional care coordinators from caregivers support groups and medical care providers.

- Developing design concepts that address findings extracted from the exploratory research.

- $\quad$ Speed dating as a means to validate user needs, obtain feedback and gain perspective on the concepts.

- Physical form studies with various material forms and interactions as a way of further exploring design ideas.

- Narrowing the concepts into a single, refined solution that addresses defined design opportunities.

\subsection{Scoping}

This is the key question that guided my initial explorations: how can emerging technologies with an IoT approach empower both senior care recipients and caregivers in a way that comprehensively addresses caregiving needs such that seniors are sufficiently supported for autonomous living in their own homes?

Throughout my research, I continually redefined the territory and topic that I was investigating in order to better break down the scope of the problem space. Before diving into exploratory research, I conducted a preliminary research on medical journals, articles and blog posts. From my initial survey, I learned that countless family caregivers who take care of older adults in their own homes experience difficulties in coordinating shared caregiving for their loved ones. Despite this, little research has been done on how family caregivers can leverage technology for better 
in-home care coordination.

With this understanding in mind, I scoped down the focus of my study from home health care for seniors' independent living to in-home care coordination of family caregivers who take care of senior care recipients. This narrowed focus better allowed me to target specific stakeholders and build specific leading questions for in-depth interviews and needs validation.

\subsection{Exploratory Research Process}

\subsubsection{Competitive Analysis}

I wanted to reduce the risk of developing a product or system for a problem that was already solved. Thus, I chose to conduct a competitive analysis in order to explore the state of the art products and services that currently support in-home care coordination for family caregivers.

The existing services were selected for competitive analysis, based on their diversity of focus as well as their popularity. I assessed each service from the end user's point of view, and investigated customer reviews of the service for portions that I could not experience directly.

\subsubsection{Literature Review}

In order to capture meaningful insights from previous projects, I reviewed a range of published academic research from both design and $\mathrm{HCl}$ communities on a range of related topics in order to distill relevant information.

Specifically, I selected research and literature for the review according to their relevancy and credibility. The literature review included a diverse range of related works, such as Home Health Care, Caregivers, Smart Home, Ubicomp, amongst others.

\subsubsection{Fieldwork}

I wanted to gain more insight about the lived experiences of informal caregivers in managing and coordinating care activities together to care for their loved one, in order to probe for more details on the different breakdowns that might revolve around their in-home care. To address these needs, I chose to conduct a qualitative fieldwork, which involves meeting and speaking directly with stakeholders.

16 in-depth interviews were conducted with individuals of various roles, including family caregivers, care recipients, and professional care coordinators. Thanks to 
assistance from UPMC Hillman Cancer Center and Area Agency on Aging, I was able to recruit family caregivers and care recipients for my fieldwork. Forward Shady Apartments also played a significant role in recruiting senior care recipients for interviews.

In the interviews, I asked family caregivers and seniors about their current in-home care experience, especially about their routine care activities, use of artifacts and tools for accomplishing these care activities, and types of potential breakdowns in routines. I also asked professional care coordinators about their strategies for handling with difficulties and breakdowns in informal care coordination.

\subsubsection{Synthesis}

After completing my exploratory research phase, I synthesized the data by creating affinity diagrams and conceptual models. These helped me to extract insights and clarify potential implications from the research and distill the data into concise findings.

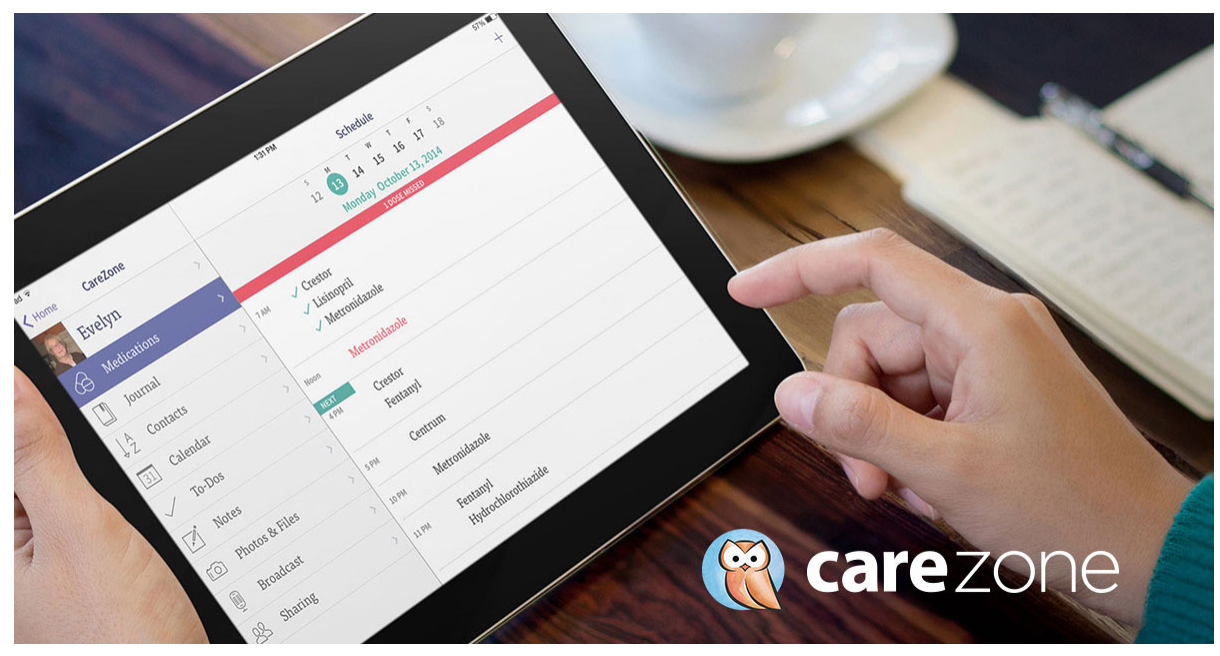

Fig. 1 Competitive Analysis Case \#1 CareZone (http://carezone.com/home)

CareZone makes it easier to take care of oneself and other family members by allowing them to organize and share important information, such as medical appointments, prescriptions, contacts, and etc. Its focus is on caregivers' access to the necessary information to keep everything organized and coordinated with other caregivers. It also partners with healthcare providers and non-profit disease organization to assist with caregiving needs of each organization's stakeholders. 

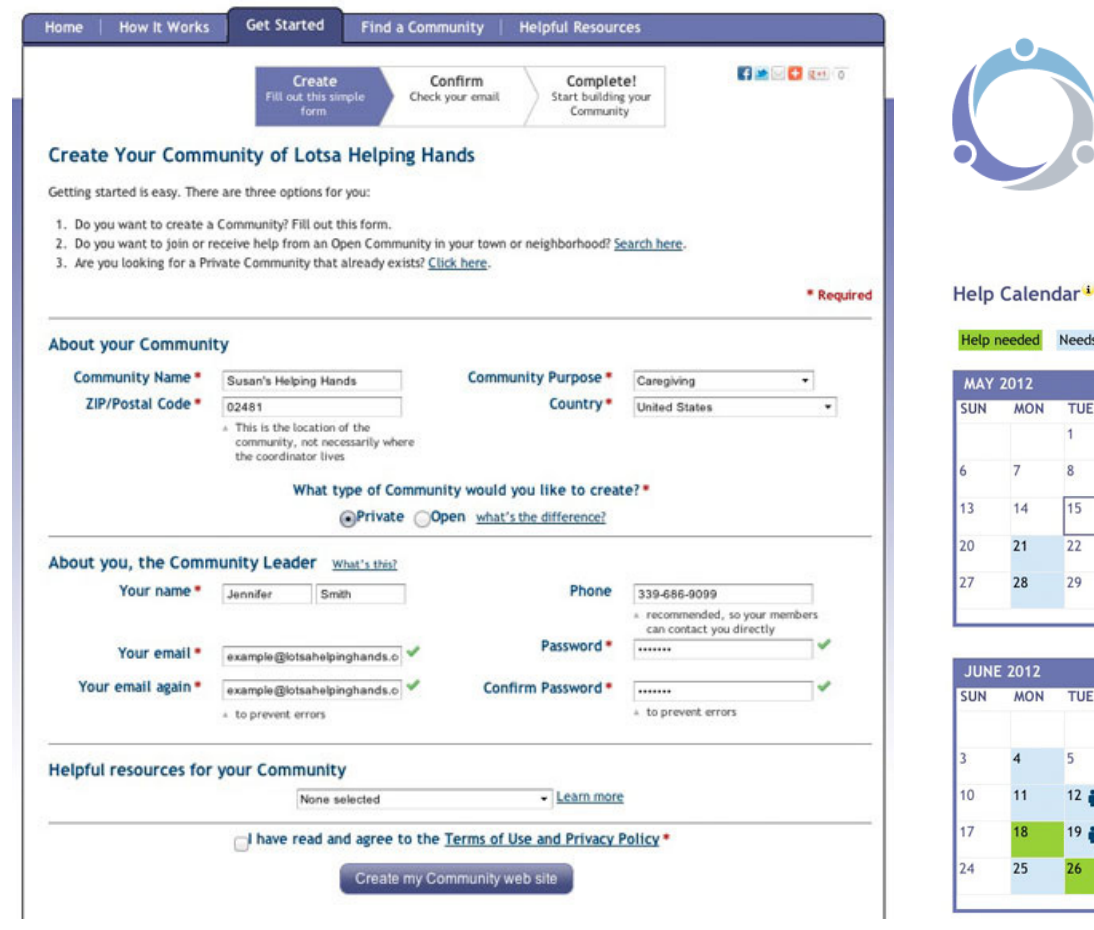

\section{Lotsa Helping Hands \\ CREATE COMMUNITY}

Help Calendar ${ }^{\mathrm{i}}$

Help needed Needs met Occasions im scheduled
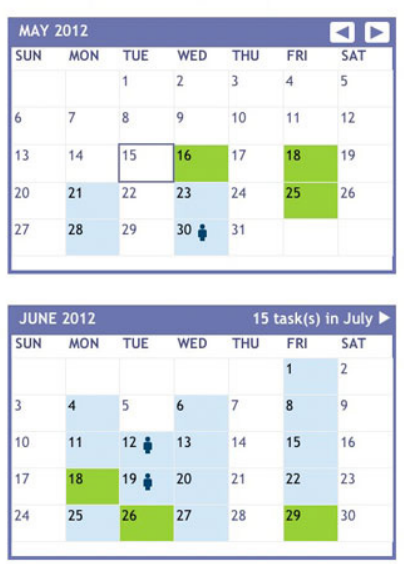

Fig. 2 Competitive Analysis Case \#2 - Lotsa Helping Hands (http:// lotsahelpinghands.com)

Lotsa Helping Hands allows users to easily create informal care network and better communicate with each other within the network of care. It focuses on internal communication within the network of care and the sense of solidarity. By providing a way to create an informal care network, it enables users to coordinate care and organize help in an easier way. Users can post requests for support needed so that others within the community know what they need. It also encourages informal caregivers to connect with each other to keep them informed and engaged.
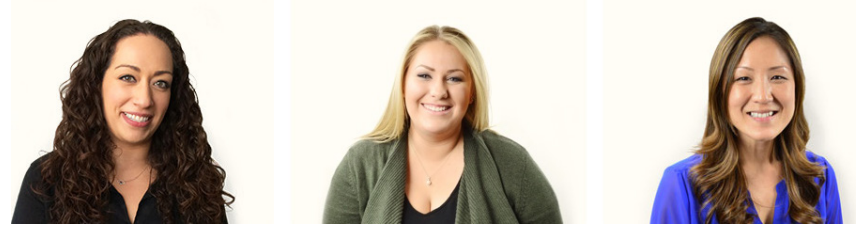

Fig. 3 Competitive Analysis Case \#3 Honor (http://joinhonor.com)

Honor is a service that connects professional caregivers with patients and their families. Care professionals visit families' home to care for seniors who want to stay in their

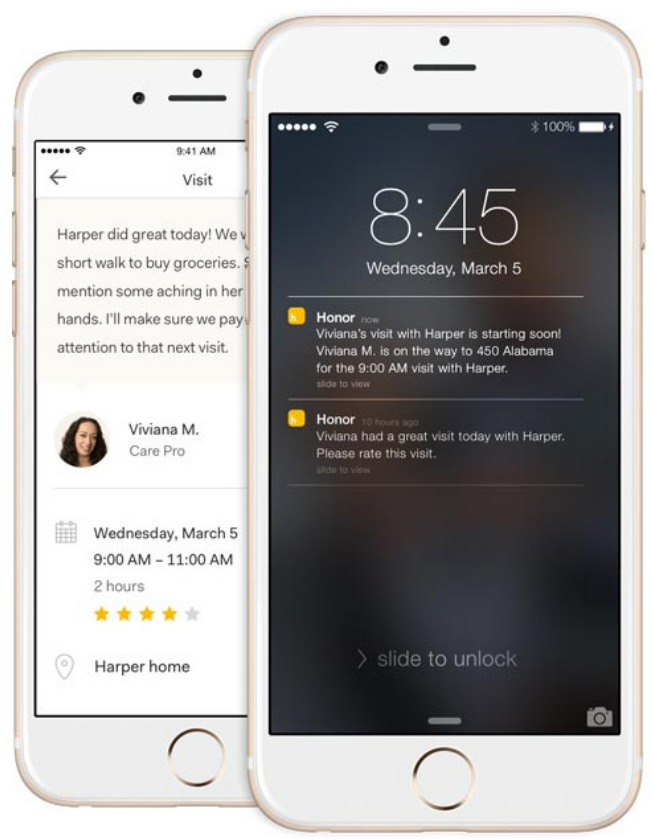


own homes. They do everything for seniors to help them live their lives, such as cooking meals, assisting with dressing and bathing, and etc. While the caregiver is at home caring for the care recipient, families get informed of real-time information of caregiver's activity. Families are shown who takes care of their care recipient, how long the caregiver is at home, and what activities they do.
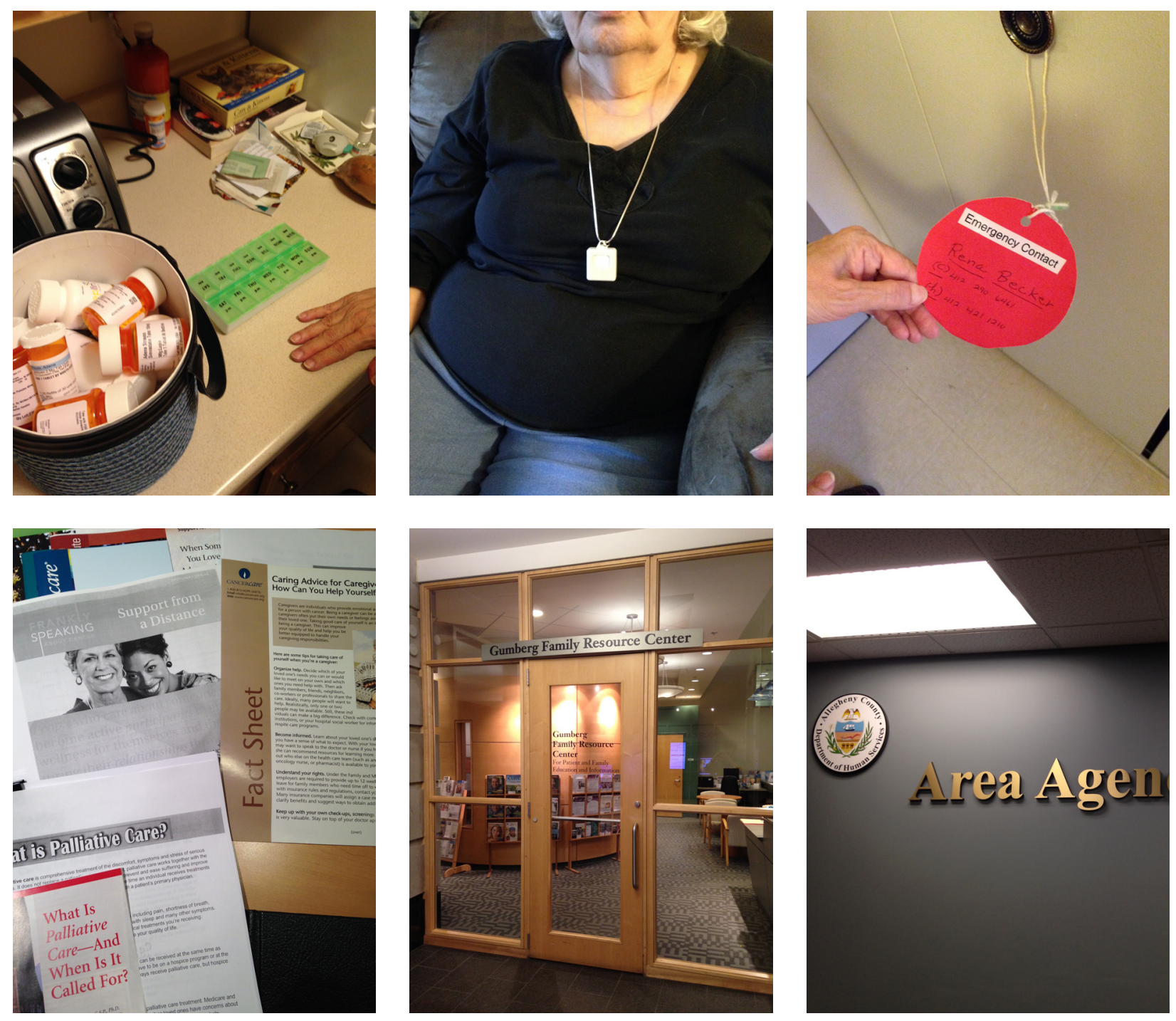

Fig. 416 in-depth interviews were conducted with family caregivers, care recipients, and professional care coordinators from UPMC Hillman Cancer Center, Area Agency on Aging, Longwood at Home, and AARP Hawaii. 

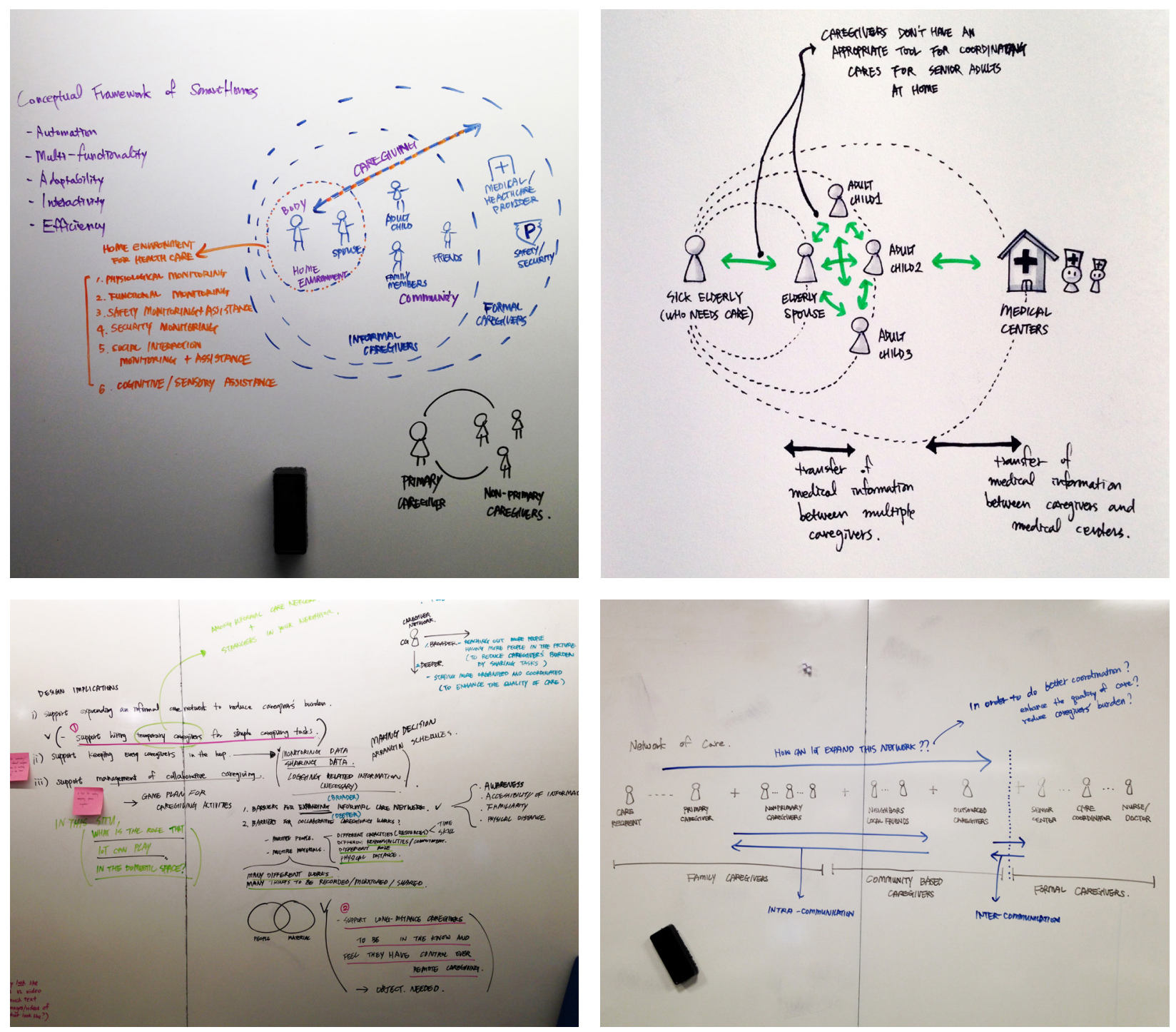

Fig. 5 Affinity diagrams and conceptual models for synthesizing the findings from the exploratory research (Top row: Conceptual models of territories of informal care network, Bottom row: Affinity diagrams of dynamics of responsibility and role of informal caregivers) 


\subsection{Exploratory Findings}

\subsubsection{High-level findings}

Exploratory research resulted in four key sets of observations.

"The challenge was definitely that I had to do lots of work. It was sometimes overwhelmingly stressful." (P3 - Primary caregiver)

1. Caregiving for loved ones is a set of specific duties that span a wide range of emotional and logistical responsibilities. Because it requires not only a lot of time but also a diverse set of responsibilities, which can be very stressful, family caregivers are likely to be overwhelmed by what they have to do. To balance work, life, and caregiver role is an uphill task. Based on my fieldwork, I clustered informal caregivers' care activities and responsibilities into four main categories to further understand their challenges in their day-to-day lives.

\begin{tabular}{|c|c|c|c|}
\hline Medical Care & Emotional Care & Assistive Care & Self Care \\
\hline $\begin{array}{l}\text { Managing loved } \\
\text { one's treatment }\end{array}$ & $\begin{array}{l}\text { Providing emotional } \\
\text { support }\end{array}$ & $\begin{array}{l}\text { Helping practical } \\
\text { matters }\end{array}$ & $\begin{array}{l}\text { Taking care of } \\
\text { oneself }\end{array}$ \\
\hline $\begin{array}{l}\text { Giving medicines } \\
\text { Monitoring vital signs }\end{array}$ & $\begin{array}{l}\text { Being with and listening } \\
\text { to the loved one }\end{array}$ & $\begin{array}{l}\text { Arranging schedules } \\
\text { Financial management }\end{array}$ & $\begin{array}{l}\text { Taking a regular respite } \\
\text { Joining a support group }\end{array}$ \\
\hline
\end{tabular}

Fig. 6 Four main categories of informal caregiver's care activities

Fig. 7 Caregivers and care recipient relationship model 1

CR: Care recipient PCG: Primary caregiver NPCG: Nonprimary caregiver

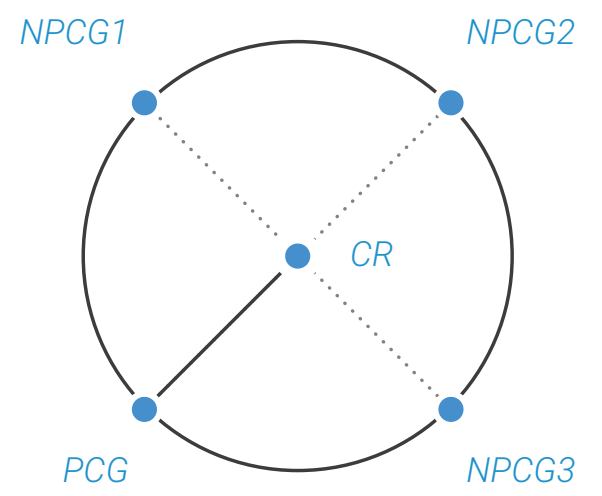

"I can only deal with errands while my sister-in-law is caring for my wife." (P7 - Primary caregiver) "To coordinate and communicate care for my elderly father with my siblings requires endless phone calls and emails." (P3 - Primary caregiver)

2. Family caregivers can reduce their individual burden by sharing care tasks with each other. One of family caregivers that l've talked with is a full-time caregiver for 
his wife who has dementia. Because his wife needs constant care and supervision, he can only run errands while another caregiver is at home and cares for his wife. By widening their care network and spreading the work, they can reduce this burden. However, as they increase their informal care network, they increase a coordination cost. Therefore, solely growing the care network and distribution of care tasks do not necessarily always reduce overall caregiving burden. For example, the more people in the network, the more difficult it may be for them to make sure that everyone is updated with recent information. To coordinate and communicate care with multiple family caregivers remains challenging.

Fig. 8 Caregivers and care recipient relationship model 2

CR: Care recipient PCG: Primary caregiver NPCG: Nonprimary caregiver

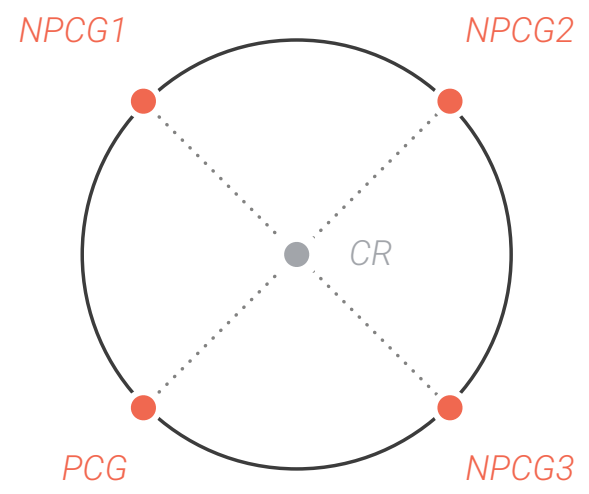

"Sometimes I'm not quite sure if I should do something for my dad while I'm caring for him because it's unclear whether somebody else has already done it." (P8 - Nonprimary caregiver)

3. Communication within the network of care is a crucial part of the family caregivers' care coordination as they have to stay informed and coordinated to maintain the quality of care. Caregivers sometimes have trouble with figuring out which specific care activities they should undertake for the care recipient, because it's not always clear if someone else has already done those tasks. Poor communication among family caregivers when they work together can result in a lack of visibility in care provision status, which results in reduced quality of care. Specifically, this presents itself in two different types of breakdowns: i) overlapping care - two people show up to do same thing, ii) forgetting care - no one shows up to do anything because they are all assuming somebody else will. In order to prevent ambiguity and failures in clear communication, informal caregivers develop their own coping strategies within their daily routines of care. For example, one of the family caregivers that I met said that she places the pill container on the table to indicate that her mom hasn't taken medications yet, so someone needs to make sure to giver her medicines. If someone in her family brings her mom medications, he or she has to place the pill container somewhere else to indicate that her mom has already taken a particular medication. In this case, her family has developed their own strategy for collective care, which uses a specific object and location as a physical/visual marker to signify a completion 
status of specific care activity.

Fig. 9 Caregivers and care recipient relationship model 3

$C R$ : Care recipient PCG: Primary caregiver NPCG: Nonprimary caregiver

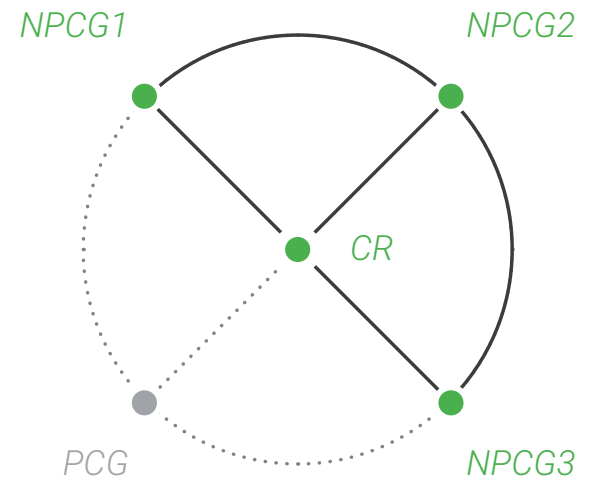

"I wish I could visit him at least once or twice a month. It's hard to keep up to date with all the medical information that changes frequently." (P4 - Nonprimary caregiver) "I took my dad to the doctor the other day, and I was asked a bunch of questions that I couldn't answer. I felt so helpless." (P5 - Nonprimary caregiver)

4. Nonprimary caregivers experience difficulties in keeping up to date with crucial information because it is complicated and changes frequently. As they don't provide care directly for the care recipient on a regular basis, it's even harder for them to remember every important detail of care. In addition, many nonprimary caregivers can be fearful of being more of a hindrance than help, due to their lack of familiarity and experience as a caregiver.

\subsubsection{Insights and Opportunities}

Research findings revealed a key insight into the role of loT in supporting an informal care network. This allows the whole network of care to function more holistically by minimizing barriers that exist between each territory within the network.

Allowing the whole network of informal care to function more holistically by minimizing barriers that exist between each territory within the network of care

With this understanding, I identified three main design opportunities for loT to help an informal care network to better coordinate and communicate care for their loved ones:

1. facilitating care works by reminding family caregivers of tasks that need to be done and providing instructions on how to accomplish it,

2. having crucial information accessible to everyone in the network of family caregivers to stay organized and coordinated, and 
3. keeping everyone engaged and connected to avoid breakdowns caused by poor communication and lack of information.

\subsection{Generative Research Process}

\subsubsection{Concept Generation and Needs Validation}

As I moved into generative phase, I begun investigating the solution space by envisioning many possible preferred interactions of family caregivers with technologies that tackle with challenges and breakdowns family caregivers currently experience. ${ }^{1}$ In order to sketch visionary interactions, I generated 13 design concepts that address the needs I identified based on the findings from exploratory research. These design concepts also explore different types of breakdowns, interactions, and interventions of IoT technologies for in-home care coordination.

Following concept generation, I met family caregivers and professional care coordinators again to conduct needs validation session. The main goal of a needs validation session was to gain some perspective on the concept applications that I generated and to discover any unmet needs participants perceive in their own experience. ${ }^{2}$ In this session, I asked participants to look at storyboards and walked them through scenarios that describe a specific situation of informal caregiving.

\subsubsection{Physical Form Study}

Physical product form is an important element of delivering services through loT technologies. In order to investigate the solution space, I explored various object forms and the dynamic relationship between physical form and interaction. To address this challenge, I conducted a brief session of physical form study where I asked few of my classmates to actively engage with objects and envision possible interactions through different object forms. ${ }^{3}$

\subsection{Generative Outcomes}

Generative research resulted in rich information for concept development and early prototype iterations.

1 Bill Buxton. Chapter 13. Sketching Interaction. In Sketching User Experiences: Getting the Design Right and the Right Design. London: Morgan Kaufmann; 2010:136-140.

2 Davidoff, S., Lee, M. K., Dey, A. K. and Zimmerman, J. Rapidly Exploring Application Design through Speed Dating. In Proc. of UbiComp, (2007) Springer, 429-446.

3 Baskinger, M., \& Gross, M. (2010). Tangible Interaction=Form+Computing. Interactions, 17 (1), ACM 


\subsubsection{Outcomes from Concept Generation and Needs Validation}

REQUEST FOR SUPPORTS

: JESSICA (46) looks AFTER HER DAD DAN (74), WHO HAS BEEN SUTFERMG FROM RHEUMATOID ARTHRITIS.

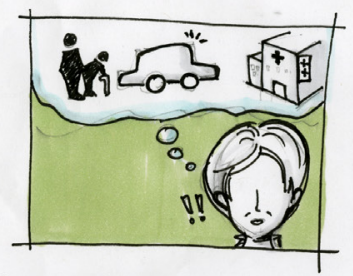

1. JESIICA NEEDS TO TAEZ DAN TO THE DOCTOR FOR A REGULAR CHECK-UP TOMORROW. SHE JUST REALIZZS THAT SHE NEEDS A RIDE TO THE MEDCAL APPOINTMENT.

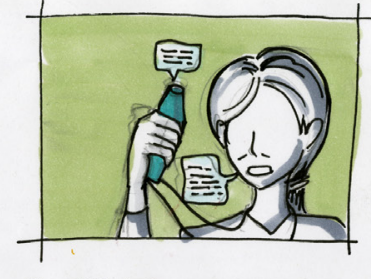

2. SHE POSTS REQUESTS FOR RIDES TO THE MEDCAL APPONTMEOT ANS MEALS FOR TOMORROW PINNER

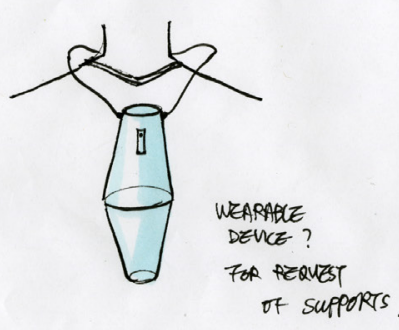

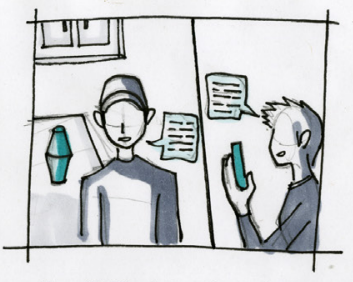

3 TOM AND RICK FROM HER NEIGHBOR VOLUNTER SUPPORTS

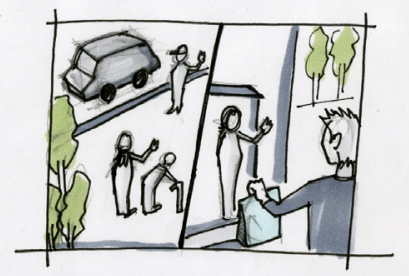

4. NEXT DAY, TOM GIVES JESILA \& DAN A RIDE To THE HOSPPTAL, AND RKE DROPS OFF THE WEAL FOR THETR DINER aT HER HOME.

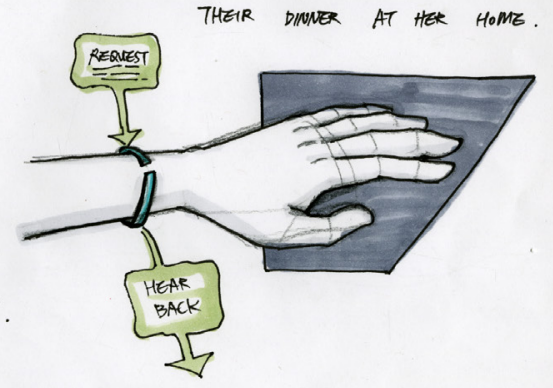

TELE-CARE SYSTEM

: ROEZRT (44) AND HIS BRUTHERS TAKE CARE of THEIR MOM, ANN(F6) WHO HAS STROEZ.

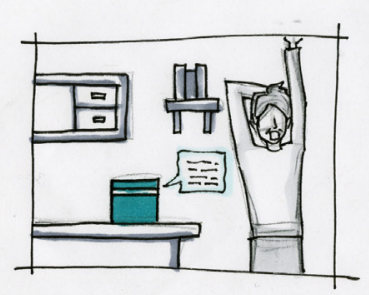

1. ROBERT WAKES WT IN THE MORNIT, AND LETS A NOTIFLATION THAT JAKE, HIS OLDEK BSETHER IS TAKING ANN TO THE DOCTOR TODAY

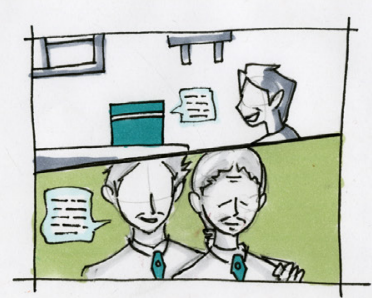

2. HE TURNS UP THE REMOTE CARE MOSE AND TALKS TO JAKE AND ANN WHILE THEY ARE heADING To THE HOSPTAL

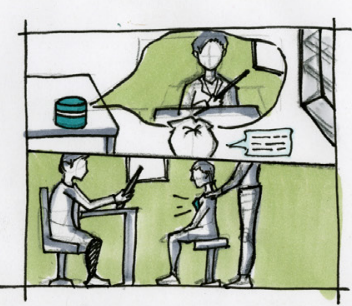

3. robzrt also can remotely ENGAGE IN THE CONVERSATION WTH DOCTOR AND USTEN TO THE DEAALS OF ANN'S HEALTH

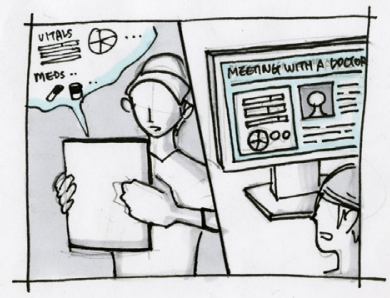

4. EVERY DETALS OF THE CONUEASATION AND INFORMATION TODAY IS ARCHIVED INTO WHERE OTHER FAMLYY MEMBER CAN ACCESS.

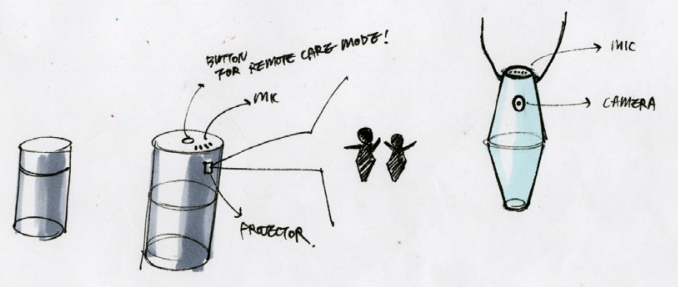




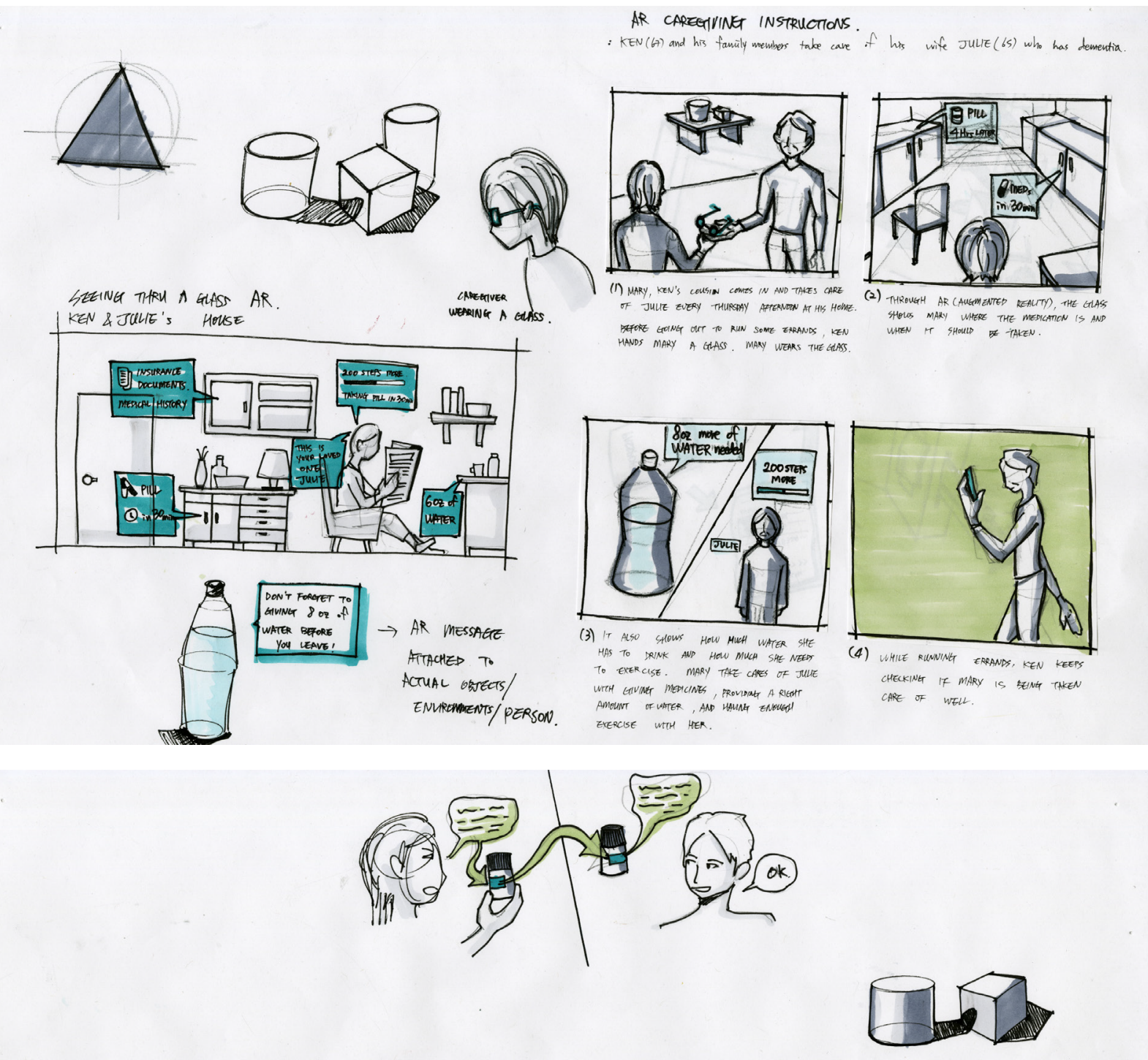

VOLCE RECORDED CARZGNING INSRRUCTIONS.

: TRACY (26) AND HER fAMIly look AfER hER GRAND FATHER KIRK(78) WHo HAS STROKE.

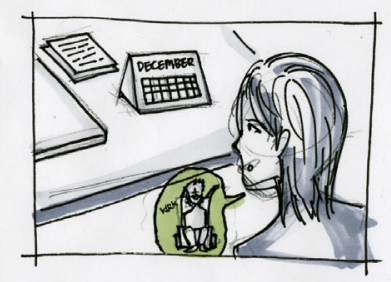

1. TRACY is GONG To Out of TOWN

For abovt A WeEk, AND SHE UANTS To

MAKE SURE THAT HER PARENG TAKE

CARE OF KTRK WTTHOUT HAVING TROUBLE

WITH MEMBSERING WHAT TO DO

WHILE SHE is AWAY.

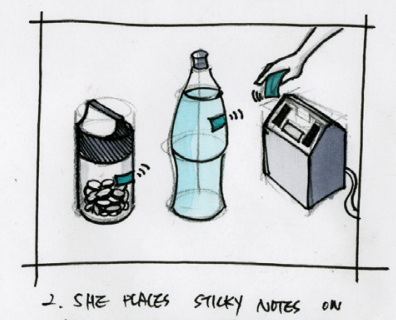

PLL CONAINERS, WATER BOTTLE,

AND OXYGEN MACHINE, ANT

ZMBEDS HER VOICE INTO THEM.

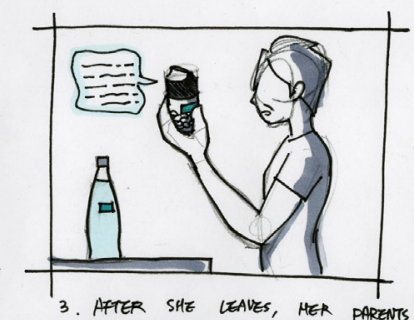

FIND A MEDICINE AND USTEN

To instructions that tracy LET

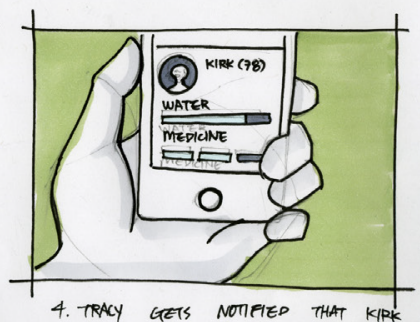

INEESTS RIGHT AMOUNT OF

MEDKINES AND WATER.

Fig. 10 Selected design concepts that address the identified needs and depict visionary interactions (Top-left: A design concept that addresses caregivers' needs of asking for supports, Top-right: A design concept that depicts receiving Augmented Reality care instructions, Bottom-left: A design concept that describes telecare system, Bottom-right: A design concept that describes updating and receiving voice instructions of care.) 


\subsubsection{Outcomes from Physical Form Study}
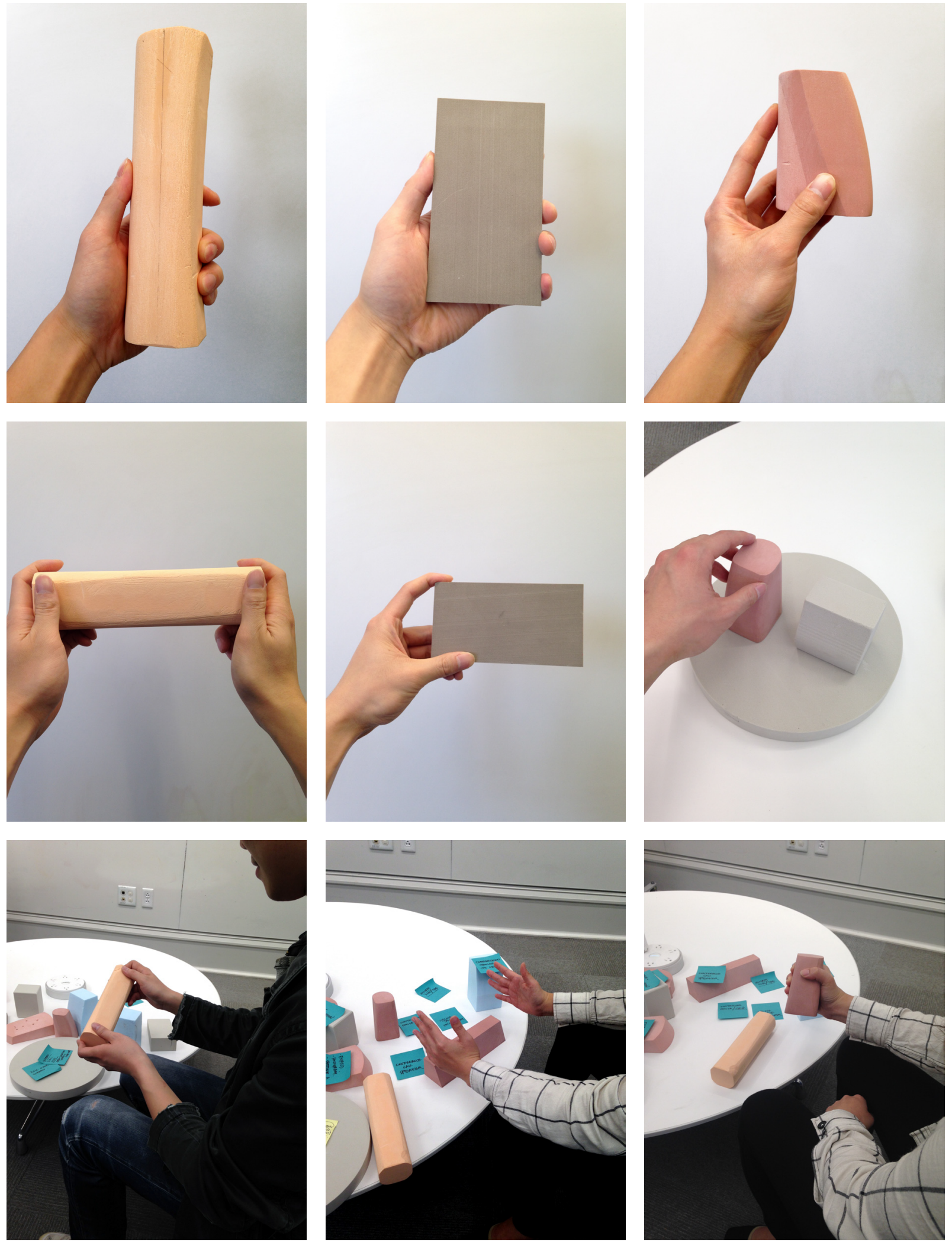

Fig. 11 Physical form study session (Top and middle row: A volumetric model of bar and controller concept, a volumetric rectangular screen, and a curvilinear volume that encourages touch and slide, Bottom row: Participants are envisioning possible interactions through different forms.) 


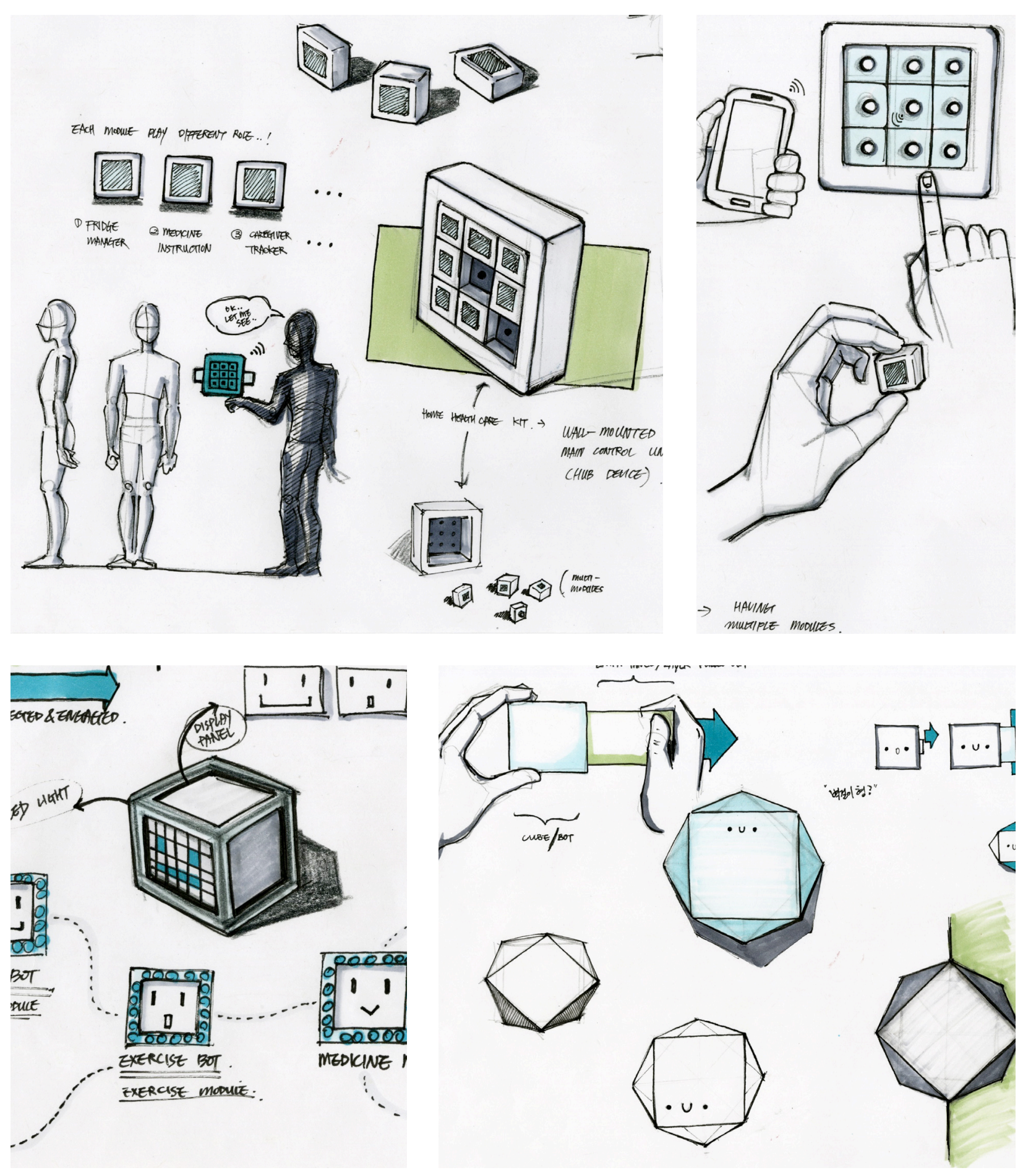

Fig. 12 Early sketches of form (Top-left and right: A concept of home care kit consisting of modularized cubes, Bottom-left: A concept of a tangible digital assistant for care coordination in a cube form, Bottom-right: A different form of a tangible digital assistant.) 

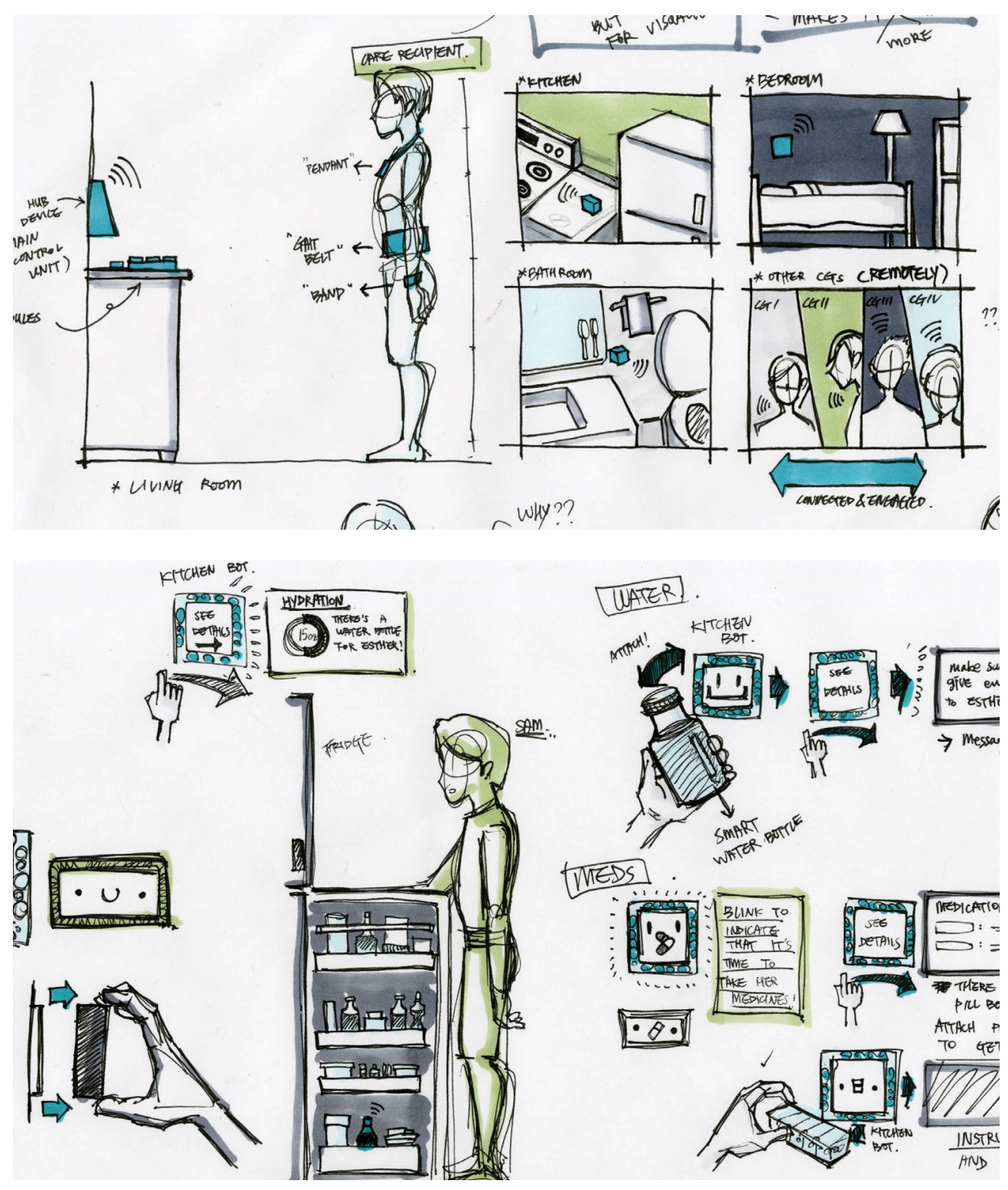

UUAER.
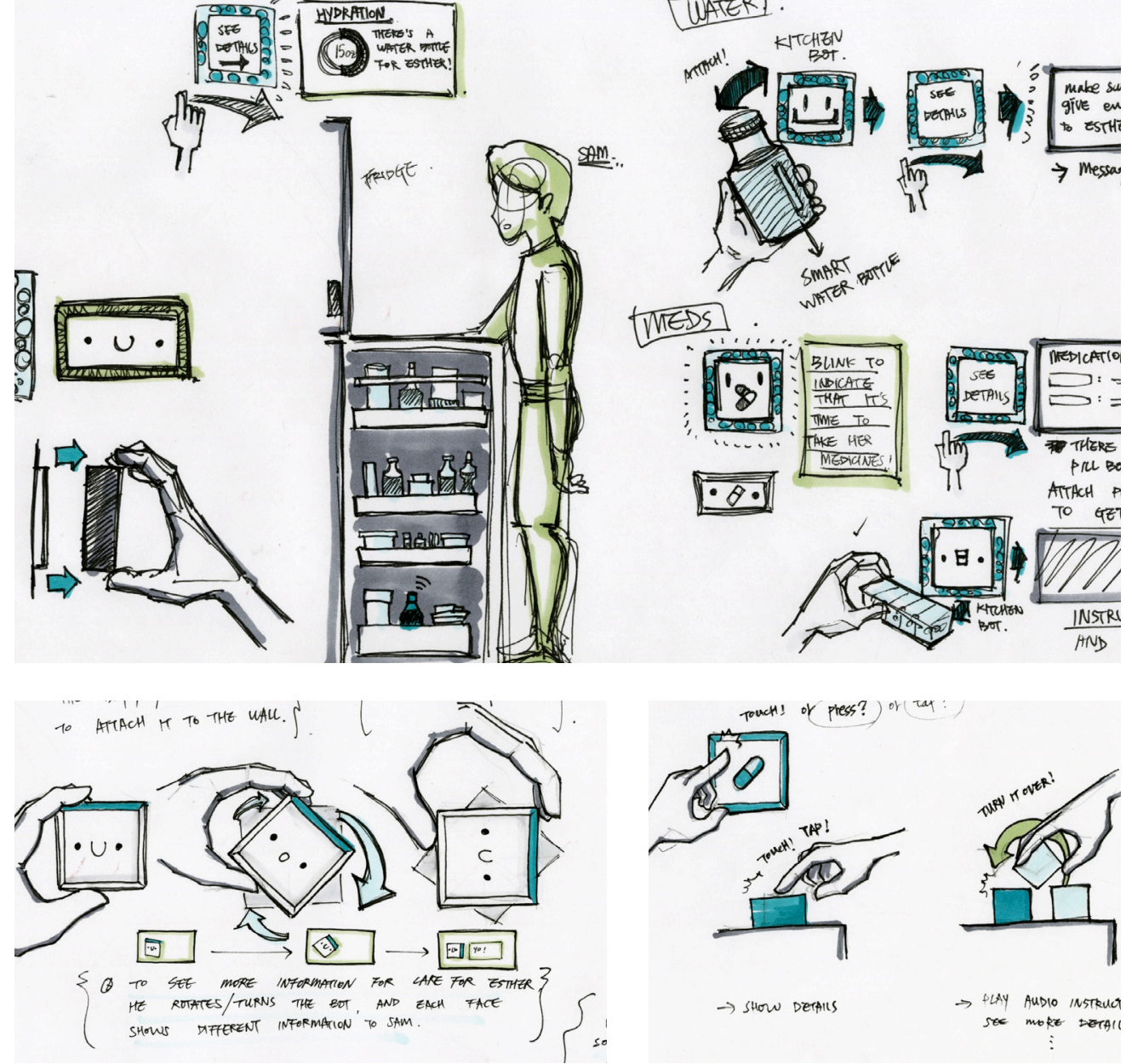

$\rightarrow$ SHow DERALS

$\rightarrow$ PLAY AUSIO INSTRUA
SEC moRE DECAII

Fig. 13 Early sketches of interaction (Top: Tangible bots for care coordination sitting in different locations in the home, Middle: Input and output of information with tangible bots, Bottom-left and right: Different ways to interact with tangible bots.) 
Fig. 14 Early prototype of

form: A physical prototype of a concept of home care kit
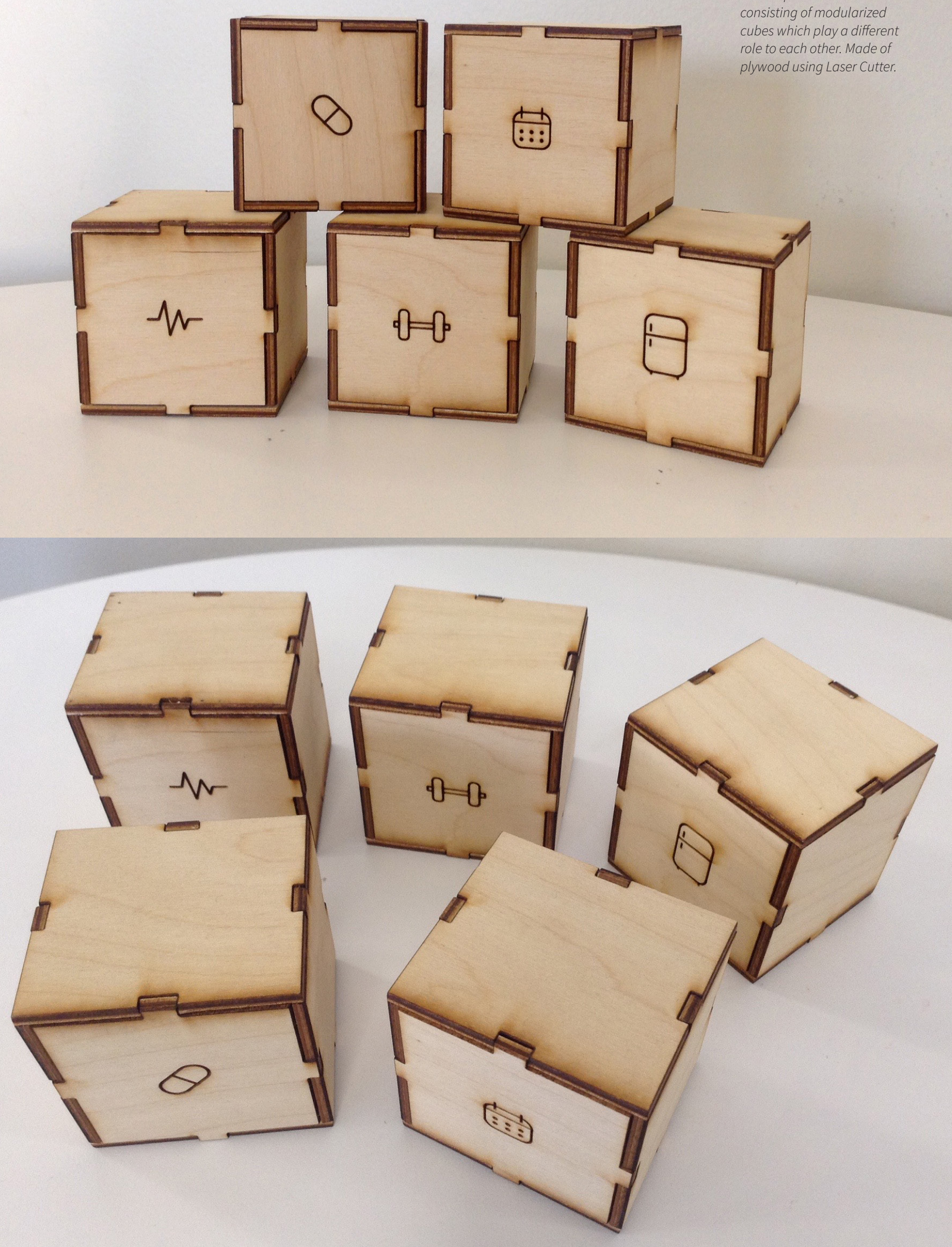
Fig. 15 Early prototype of form: Another form of

modularized cubes. Made of plywood using Laser Cutter.
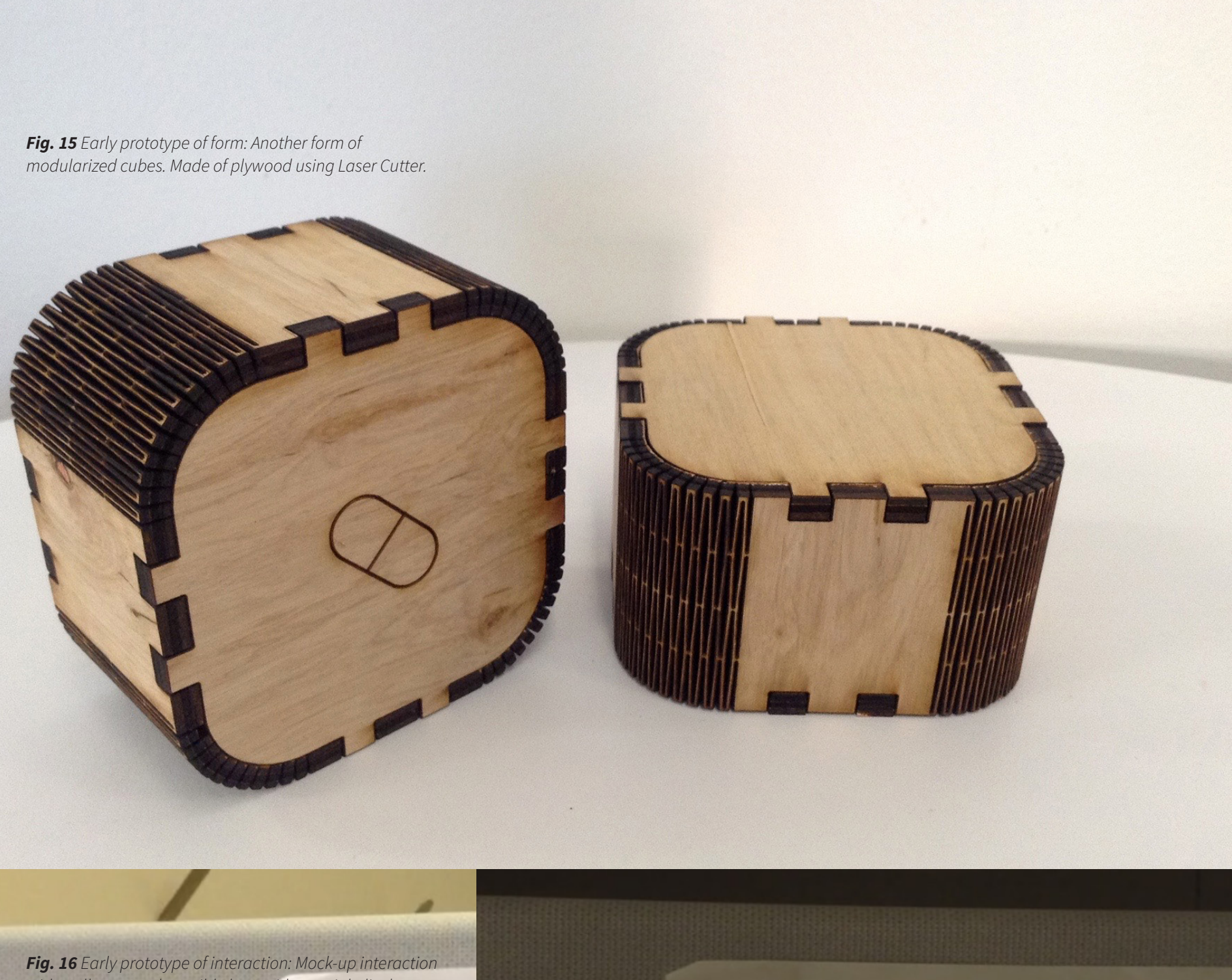

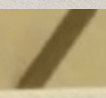

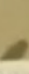

Fig. 16 Early prototype of interaction: Mock-up interaction with wall-mounted tangible bots with an e-ink display.

20

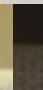

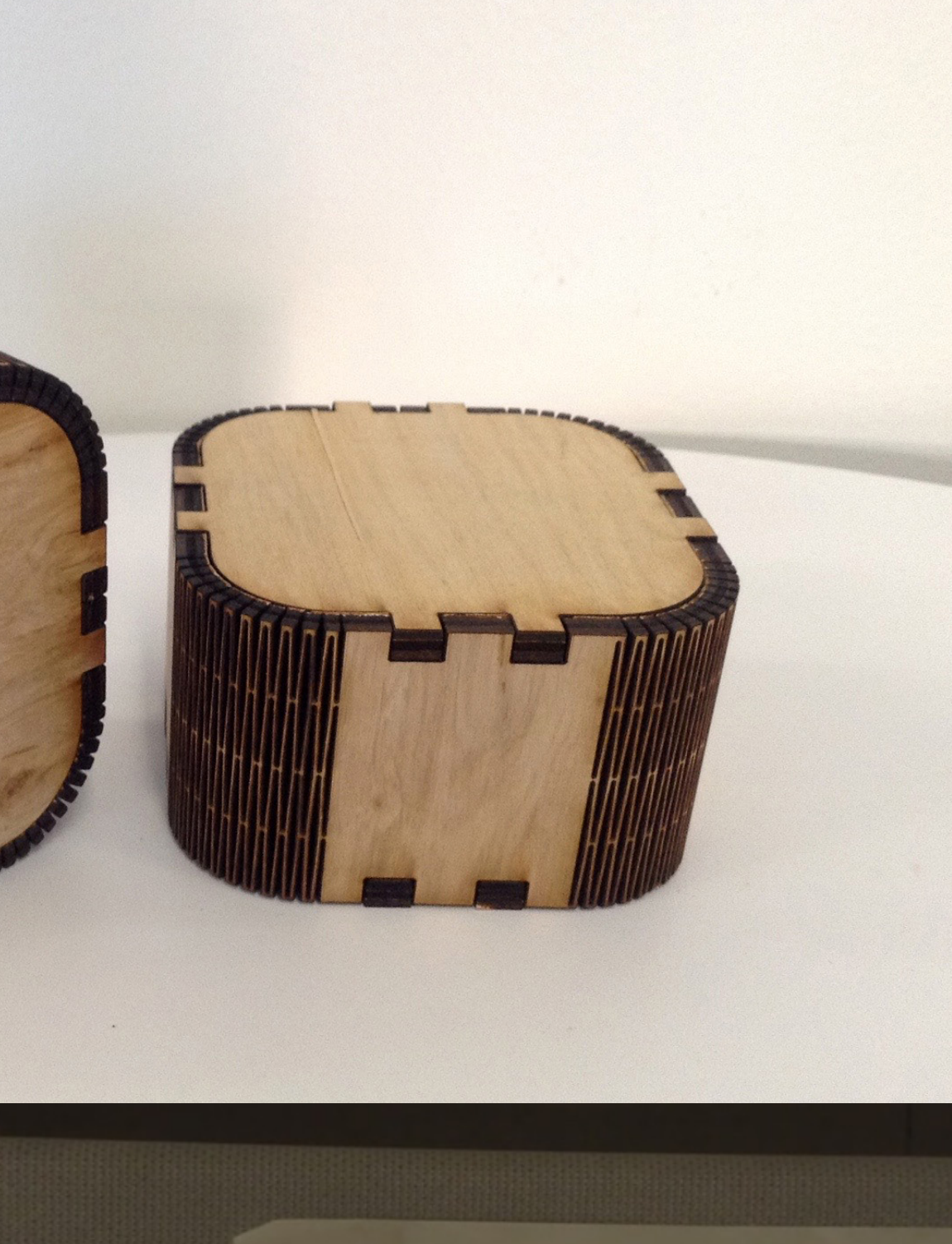

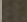


Fig. 17 Prototype of form and interaction:

Rotatable and graspable physical form
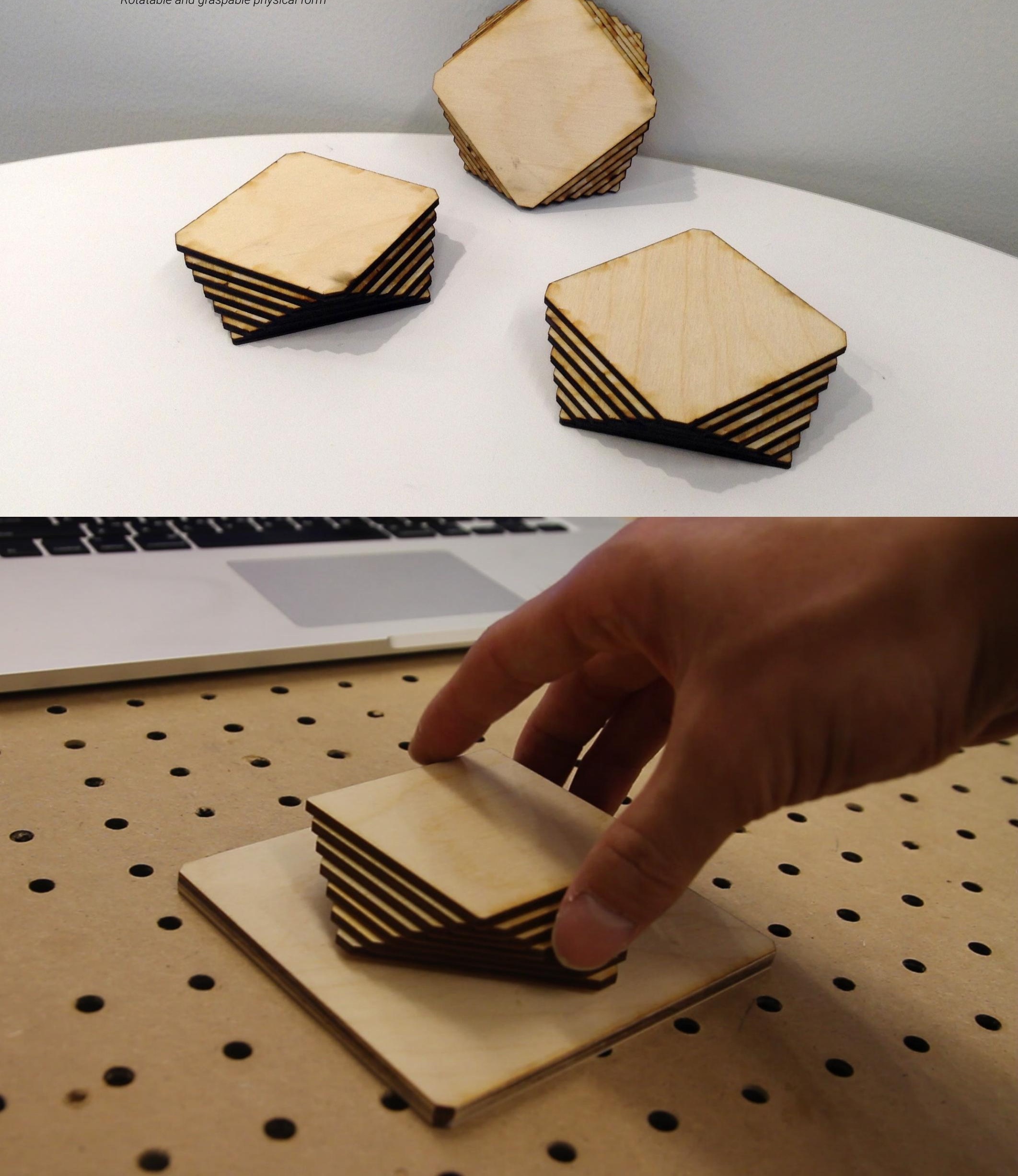
CareBot: A Smart Home Platform for Care Coordination

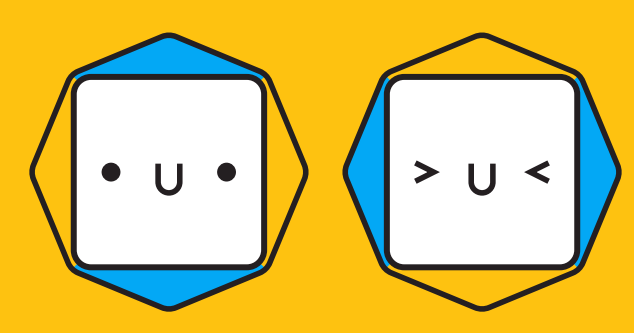




\section{Final Prototype}

\section{CareBot: A Smart Home Platform for Care Coordination}

\subsection{Concept Overview}

CareBot is a smart home platform consisting of interactive tools that help family caregivers better coordinate and communicate care for their loved one with those wishing to help them. It is designed to provide a new way of engaging in care and prevent potential breakdowns caused by having multiple caregivers who are cooperating to care for the same care recipient.

\subsection{Main Features}

Main features of the concept of CareBot are three fold; and these features specifically address design opportunities identified from qualitative research:

Remind: It triggers reminders to prompt caregivers, especially nonprimary caregivers who experience difficulties in remembering details of care, to complete care activities that haven't been done on time. It aims to reduce the chances of forgetting care works such as taking medication, drinking water, and simple exercise.

Capture: It helps caregivers capture the care activities that should be coordinated at the point of action. It provides a way to document and annotate care activities that have or haven't been done to give contextual information to other caregivers.

Connect: It keeps all family caregivers connected to crucial information of care as well as to each other. It makes it easier for caregivers to stay in the loop and organize support together. It also gives caregivers the opportunity to volunteer and take over care tasks.

\subsection{System View}

To concretize three main features of the concept of CareBot, its design should have the following system requirements. 

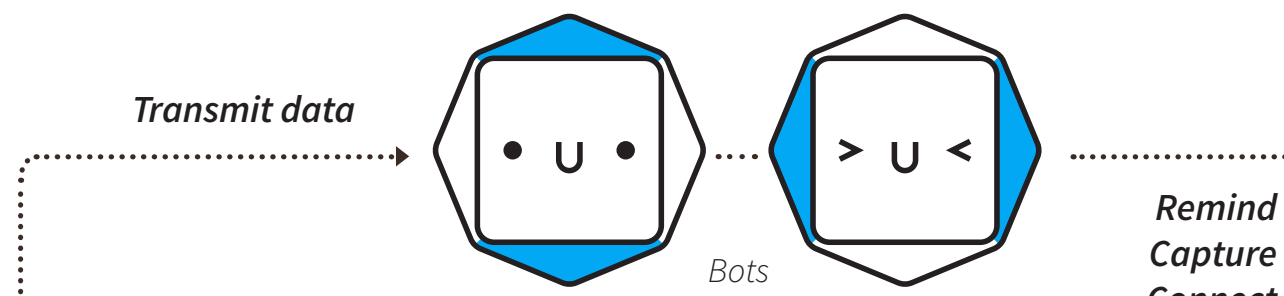

Capture

Connect

\section{Monitor \&}

sense data<smiles></smiles>

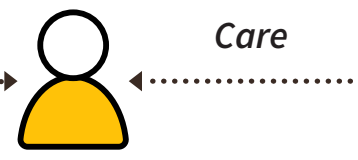

Care recipient

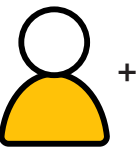

Primary + nonprimary caregivers
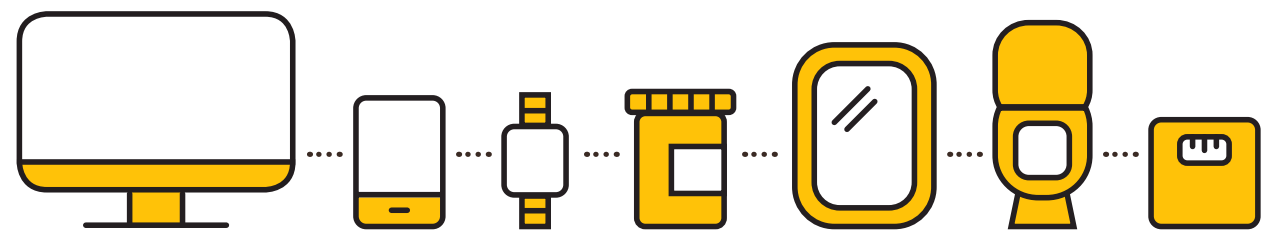

Smart objects \& devices
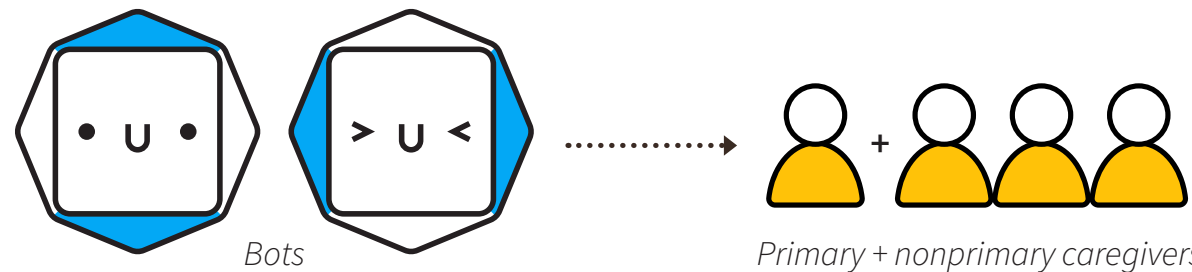

Primary + nonprimary caregivers

1 CareBot must be able to recognize each caregiver in the network when they are at home to provide more personalized instructions on care coordination. Caregivers can be identified from CareBot through using Bluetooth technologies.

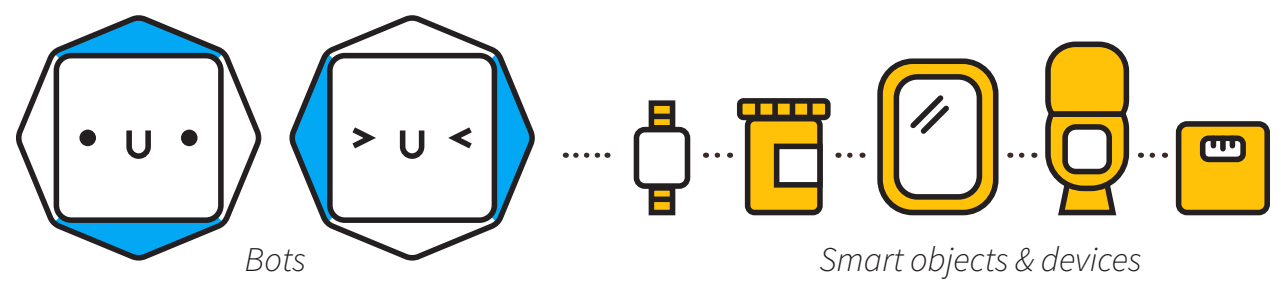

2 CareBot must understand important information of care including a regular schedule for must-do activities, medical treatment, caregivers profile, and real-time 
data of caregivers and care recipient's activities in their home.

- CareBot must communicate with a range of smart objects and devices to get necessary data of caregivers and care recipient.

- Primary caregiver must be able to input necessary information of care directly to CareBot through mobile and web application of CareBot.

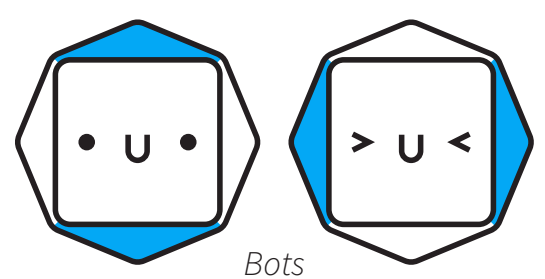

3 CareBot would need to have a physical tool directly situated in the real context of care activities to allow it to capture the care work at the point of action.

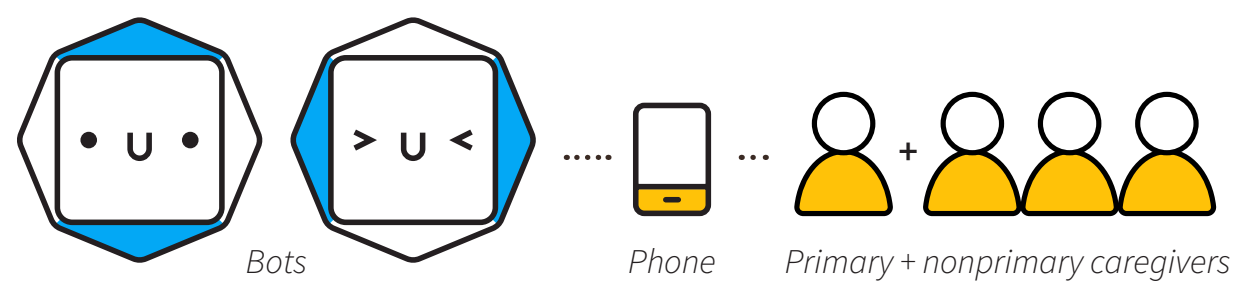

4 CareBot would need to collect and deliver messages between caregivers so that they don't all go independently to support not only more organized care plan but also caregivers' social practices.

\subsection{Scenarios}

The following scenarios depict how users may interact with CareBot within various contexts:

Users: Esther (79) is an elderly care recipient who has dementia / Lisa (48) is Esther's daughter and a primary caregiver of her / Sam (27) is Lisa's nephew and non-primary caregiver / Jason (45) is Lisa's brother and non-primary caregiver. 


\section{On-boarding}

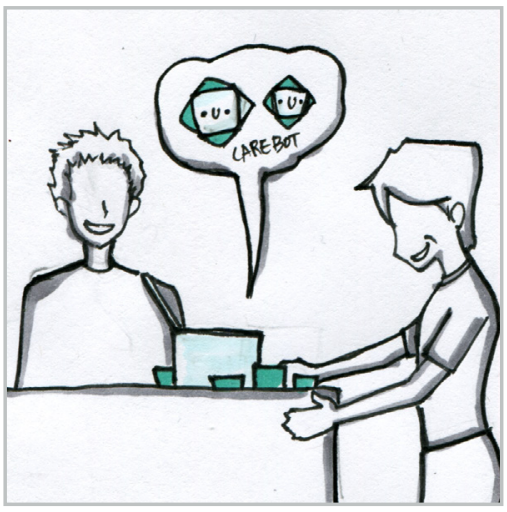

Lisa receives a gift from Jason. It's a CareBot. Jason felt that it'd be helpful for them to use CareBot to better coordinate care for Esther.

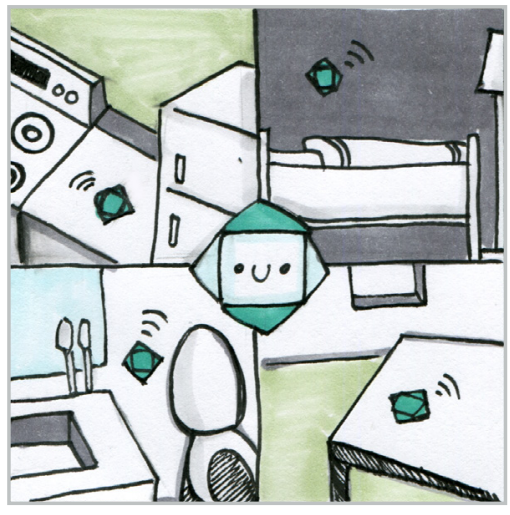

Lisa and Jason place each Bot in different locations in the home, such as living room, kitchen, and bathroom.

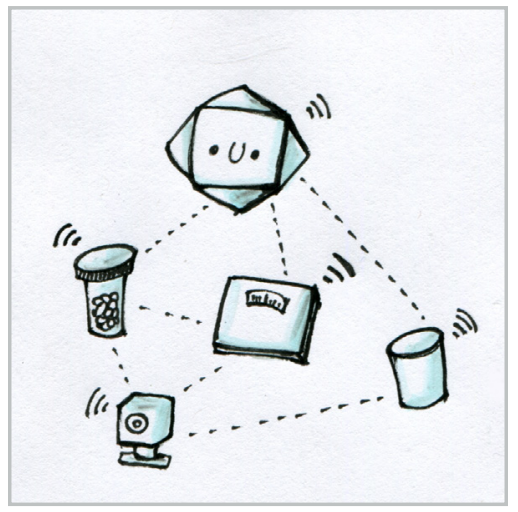

Then, they sync CareBot with other smart sensors and devices in the home, such as pillbox, scale, water bottle, and etc. Finally, they fill in details of care information through the app including profiles of family caregivers, regular schedule for must-do activities, medications, contact information and etc.

\section{Activating}

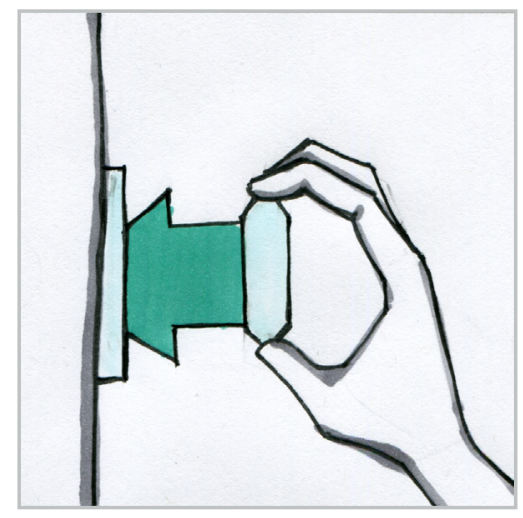

Lisa needs to go out to run some errands today. Sam is going to come and take care of Esther while Lisa is away. So Lisa activates CareBot by attaching the Bot to the living room wall.

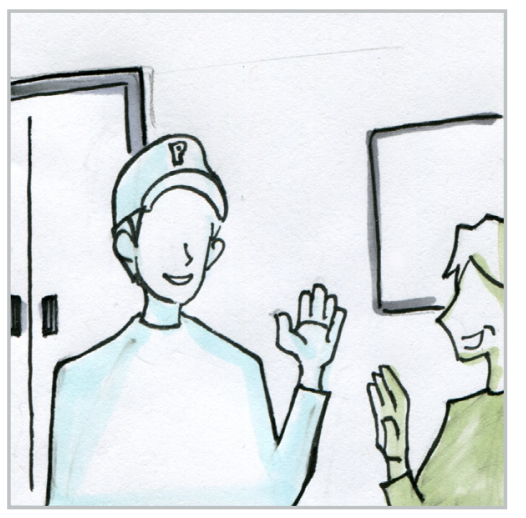

Sam arrives at Esther's house, and Lisa leaves.

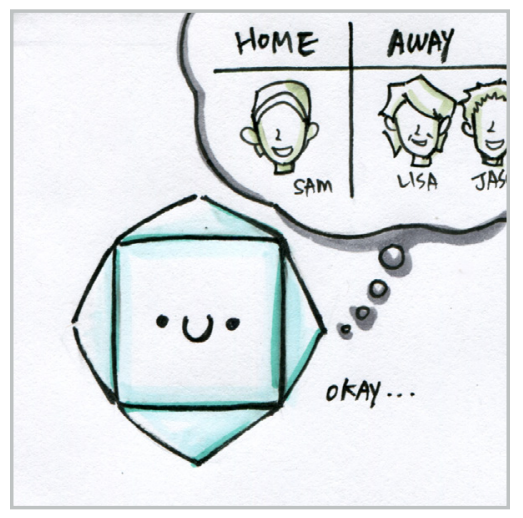

CareBot recognizes that Lisa is away now. Also, it recognizes that Sam just arrived and he needs more detailed instructions on care works. 


\section{Welcoming}

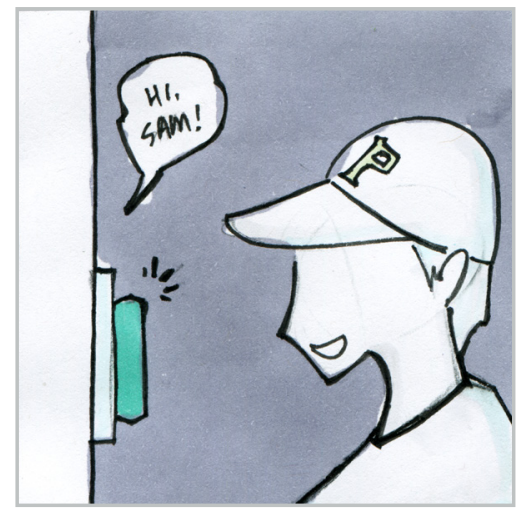

The Bot in the living room spots him and welcomes him by lighting up and showing the following message: "Hi, Sam! How are you? Thanks for your time and efforts for Esther! It has been over two weeks since your last visit."

\section{Remind}

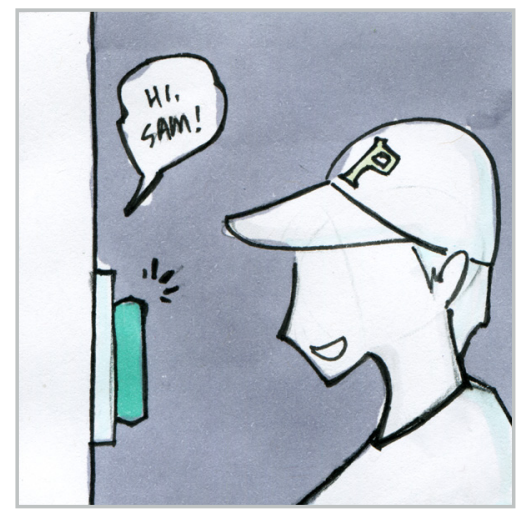

Sam goes to the kitchen, and the Bot spots him and lights up to signal that there's an action to take for Esther.
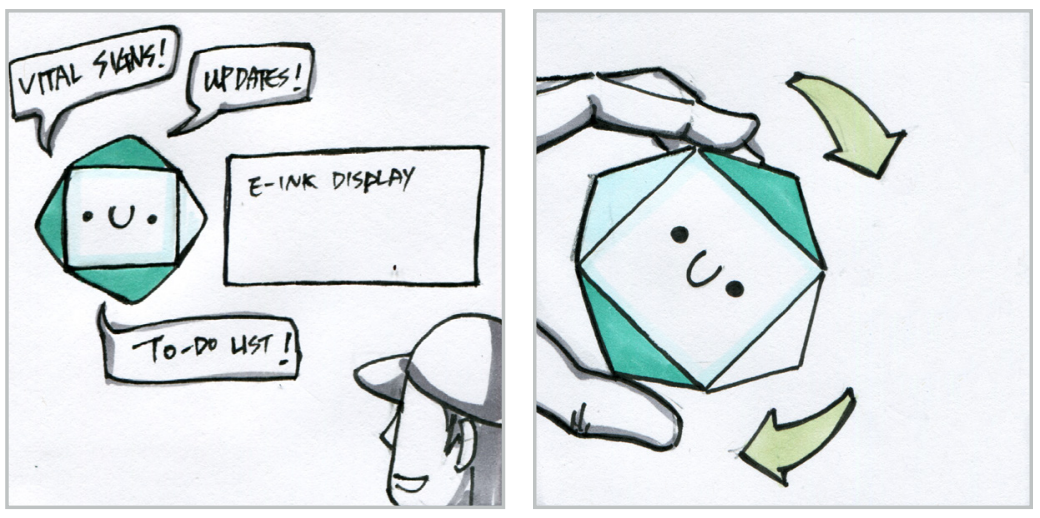

Then, the Bot glows to signal information. Each side has different types of information that Sam needs to know, such as updates since the last visit, recently monitored vital signs, and to-do list.
Sam turns the Bot, and the E-ink display shows details.

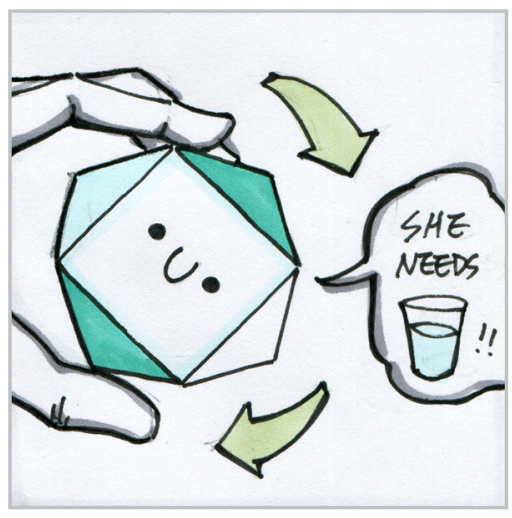

He wants to see details, so turns the Bot so the right side glows up. An E-ink display shows details: "Esther needs to drink at least 20 oz of water today, but has only consumed 6.5 oz so far."

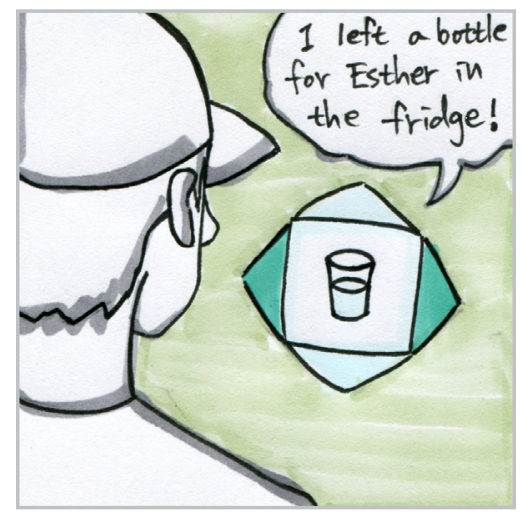

He turns the bot again to check Lisa's message about this task. She says that she's put a water bottle in the fridge with the specified amount of water for Esther. Sam tries to make sure to give Esther enough water. 


\section{Capture}

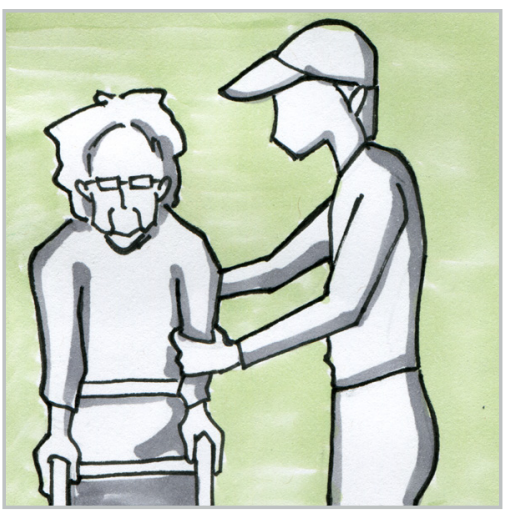

The Bot tells Sam to keep Esther moving in order for her to stay active. It recommends him to do a simple walk with her at home. Sam tries to follow instructions, but Esther doesn't seem to be in the mood for exercise.

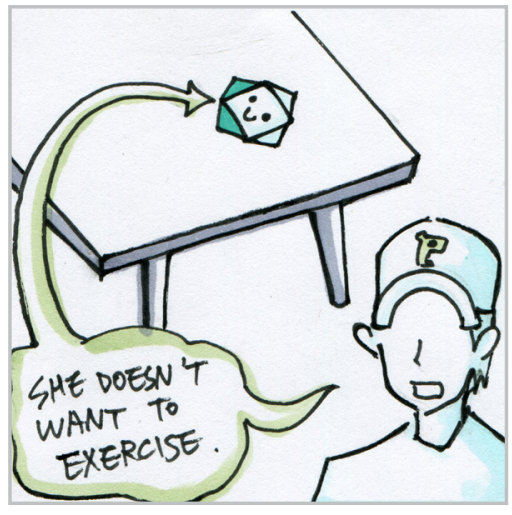

He turns the Bot and adds a note to keep other family members informed. "I tried to keep her moving but couldn't get her to.."

\section{Connect}

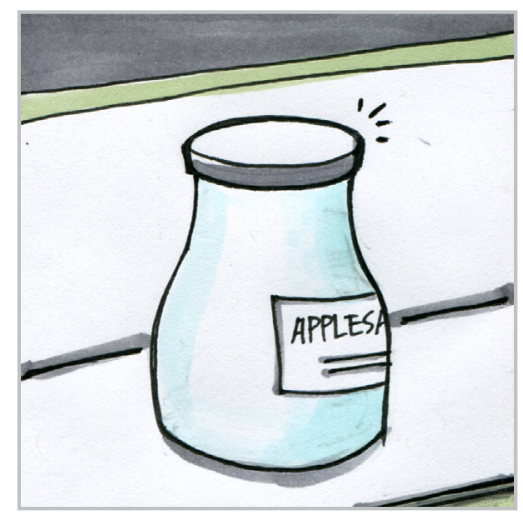

Lisa is preparing a meal for Esther. She realizes that the applesauce is almost empty. She scans the applesauce with the Bot to add applesauce to her grocery list which is shared with other family caregivers.

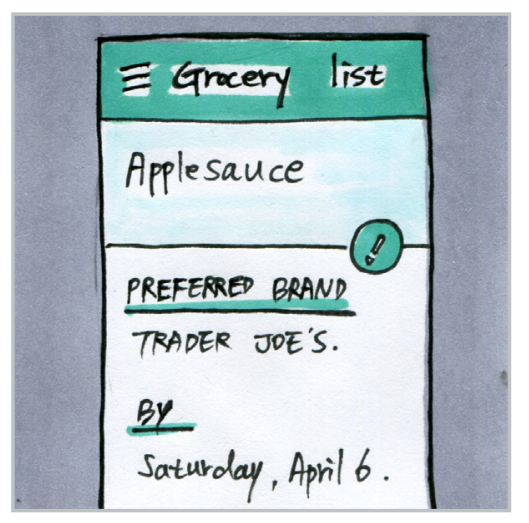

After the meal, she fills in details like when she would need it and which brand she prefers in the CareBot app.

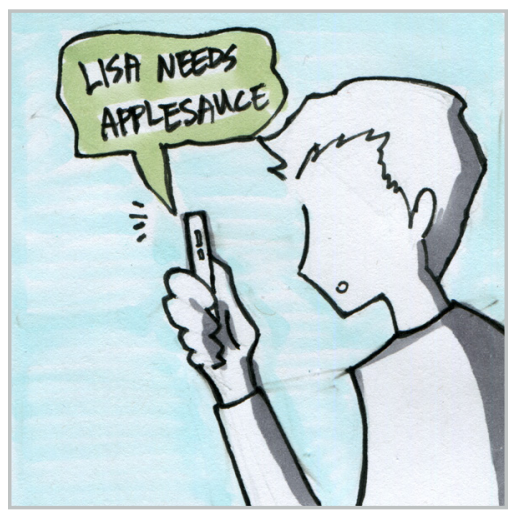

Jason gets a notification of Lisa's grocery list. Considering his next visit to Lisa's, he decides to make the trip to the grocery store in her stead. He is going to bring it to her tomorrow. 


\subsection{Design Details}

\subsubsection{Physical Form}

As discussed in System View section, CareBot should have physical artifacts that are directly located in the real context of specific activities of care to allow it to better trigger reminders and capture the care work at the point of action.

The physical tool of CareBot is comprised of two main components: the Bot and an e-ink display.

The Bot is the part that signals users to take actions and that users actually interact with to get information. It is packed with multiple sensors and components including LED display, motion detector, light sensor, camera, microphone, Bluetooth, Wifi, and etc. The e-ink display is the part that displays details of information that the Bot provides. As described in the scenarios, the Bot lights up when there's an action to take for the care recipient. Each side of the Bot has different types of information that

Each side illuminates with ambient lights

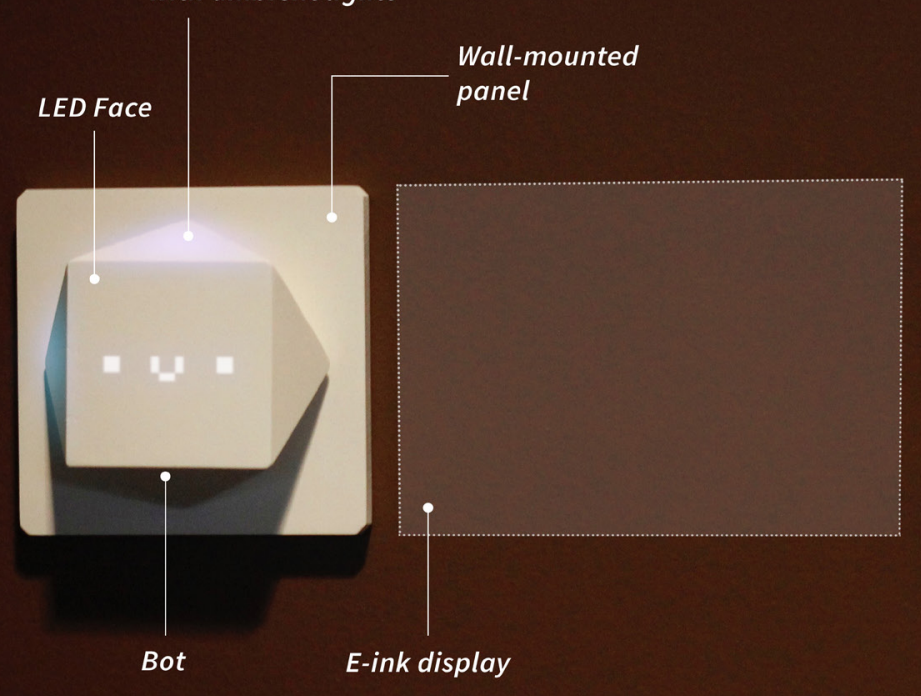

Fig. 19 The physical tool of CareBot breaks into two parts: the Bot and an e-ink display 
caregivers need to know and lights up when there's specific information to display. When users turn the Bot, the e-ink display presents details of information on the surface which correspond to the right side of the Bot.

The form of the Bot was designed in a way that it has a physical affordance which is graspable and rotatable.
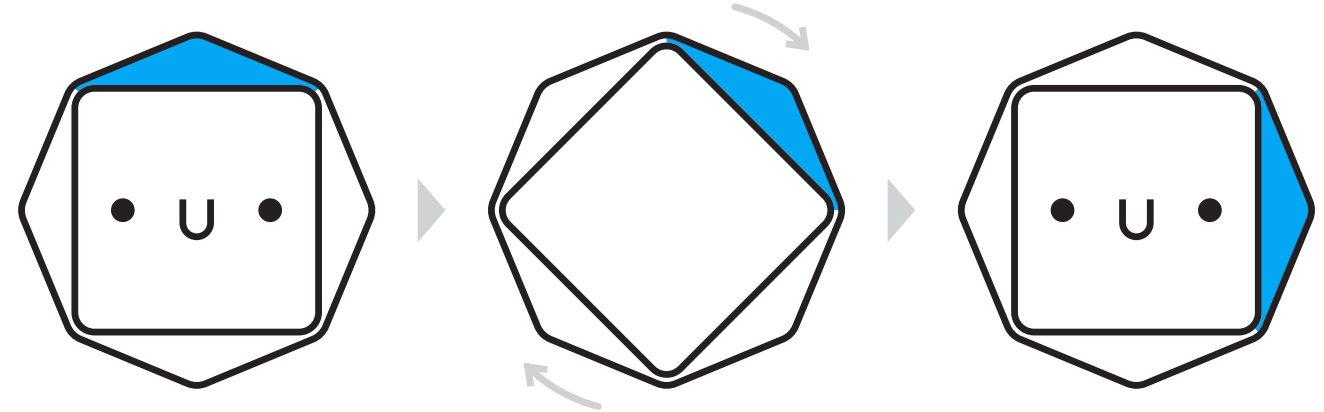

The Bot is the part that signals users to take actions and that users actually interact with to get information

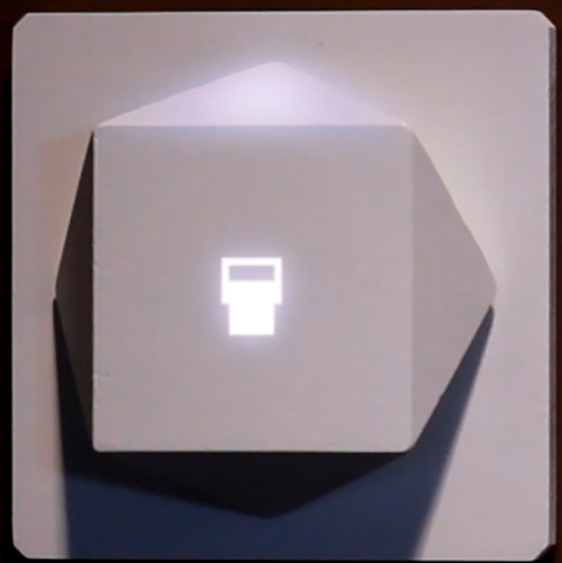

Esther needs to drink at least

20 oz of water today but has

only consumed $6.5 \mathrm{oz}$ so far 
$-\frac{10}{406}$

Fig. 21 The form of the Bot was designed in a way that it has a physical affordance which is graspable and rotatable.
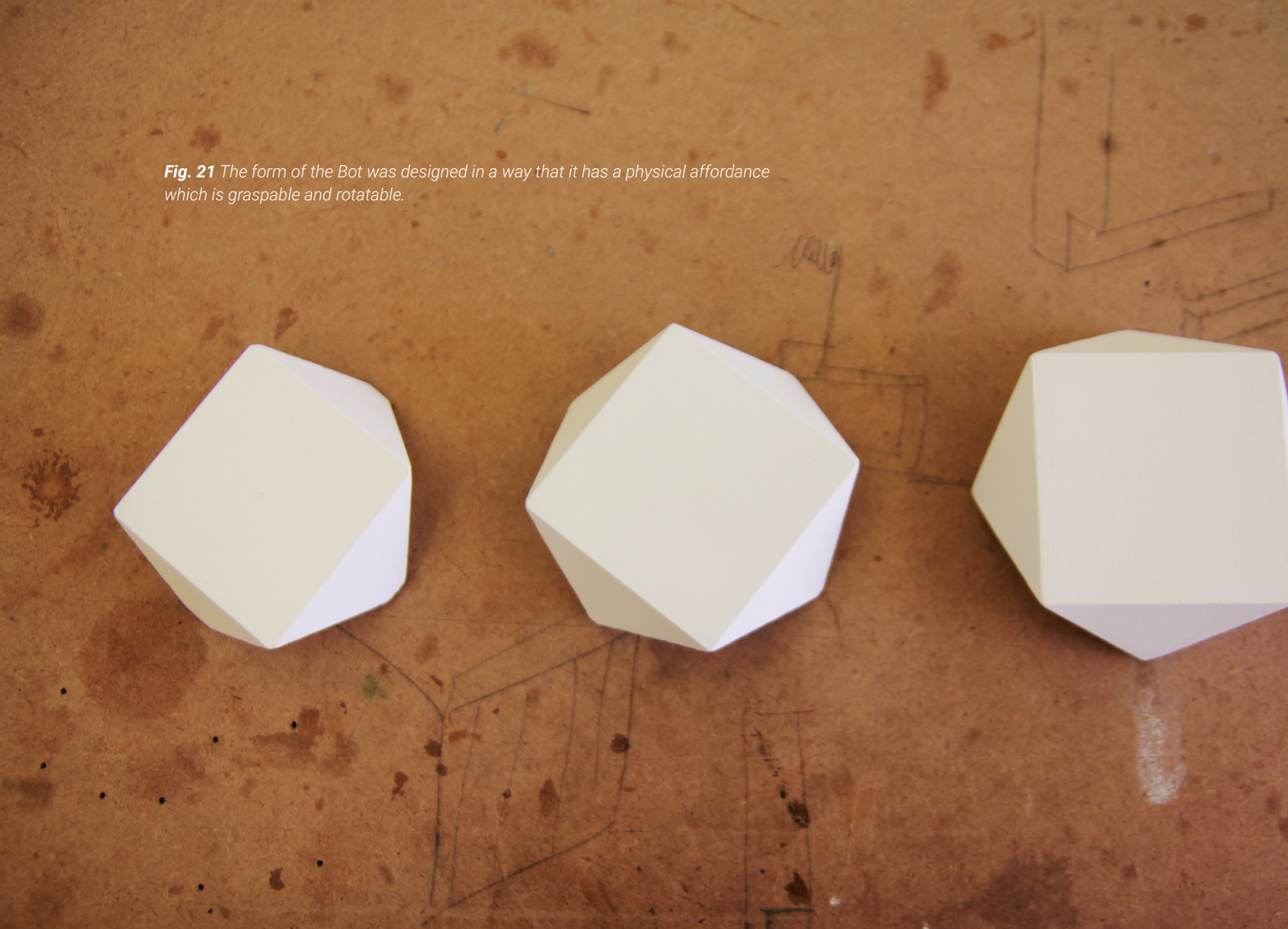


\subsubsection{Interactions}

\section{With the Bot}

Interaction with the Bot is designed to be simple and effortless to learn due to its obvious form and shape. What users do with this physical artifact is just pressing the Bot, and turning the Bot. While pressing the Bot is mainly for wake, confirm, and next, turning the Bot is for scroll and navigate.

Followings are selected procedure of interactions describing how users interact with the Bot with different goals.

\section{When the Bot welcomes caregivers}

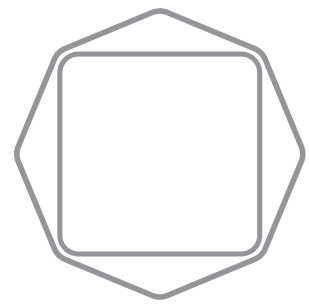

Bot

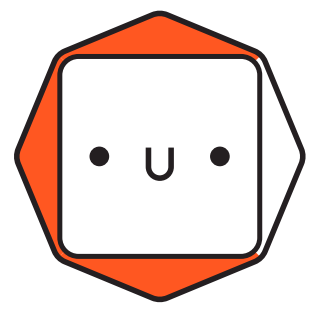

Hi, Sam! How are you? Thanks for your time and efforts for Esther! It has been over two weeks since your last visit.

\section{Sleep mode}

The Bot is waiting for caregivers in sleep mode. It keeps sensing and monitoring data.

\section{Motion detector}

The Bot welcomes caregivers by lighting up and showing a personalized welcoming message on an e-ink display when caregivers are in the room.

\section{Turning the Bot}

In order to navigate information, users need to turn the Bot so the right side lights up. 


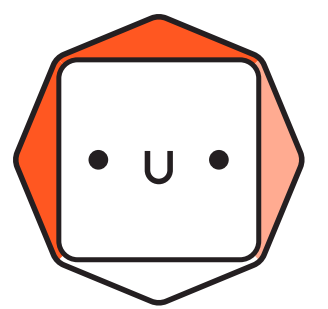

\section{Updates}

Medicines that Esther has to take

haven't been changed since your

last visit. Even if you don't

remember how to dose, no worries.

I will teach you.
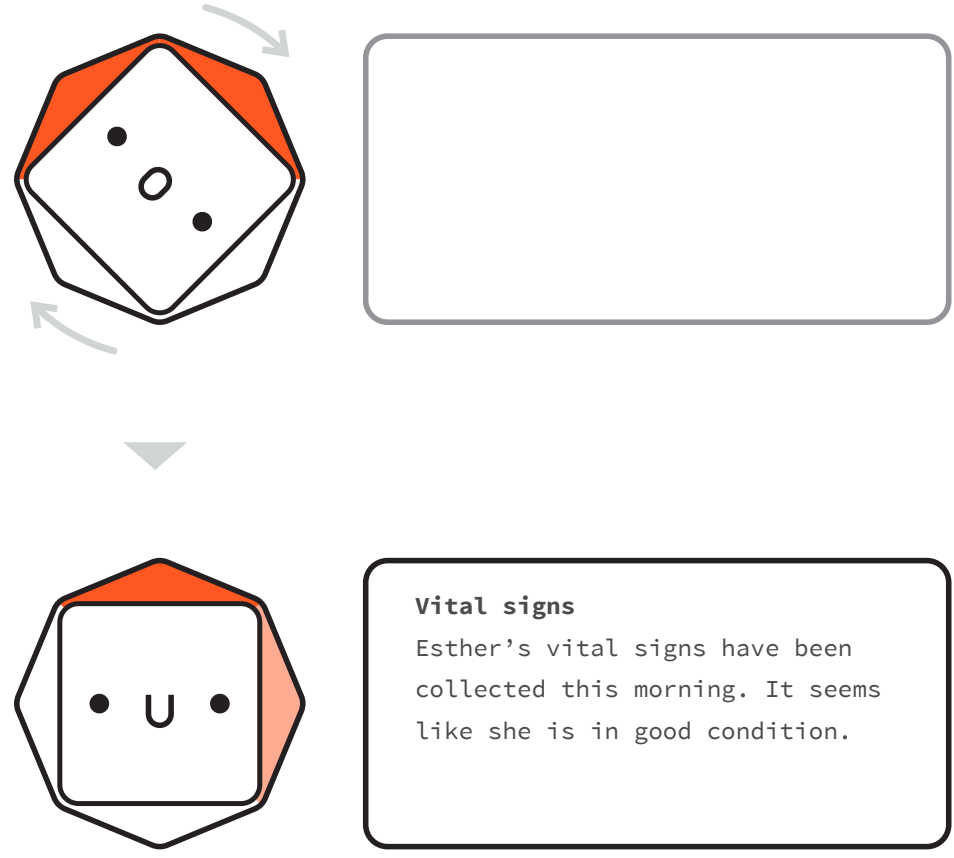

\section{Vital signs}

Esther's vital signs have been collected this morning. It seems

like she is in good condition.

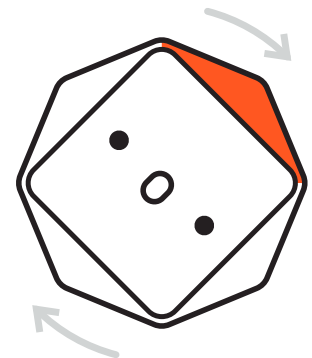

\section{Details - Updates}

The first side contains updates. It gives any updates that caregivers might need to know.

\section{Turning the Bot}

To scroll to the next information, users need to turn the Bot again so the right side lights up

\section{Details - Vital signs}

The second side is vital sings. It gives diagnostic information about vital signs and symptoms of the care recipient.

\section{Turning the Bot}

To scroll to the last information, users need to turn the Bot again so the right side lights up. An e-ink display will display the last information. 


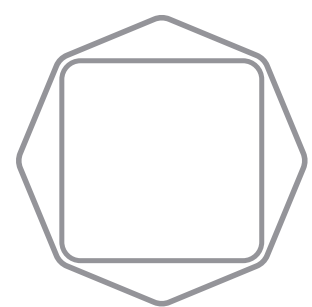

Bot
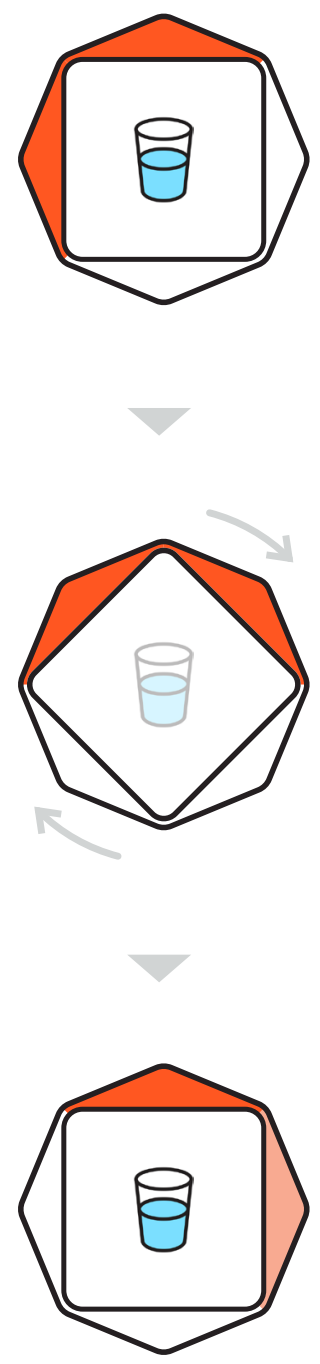
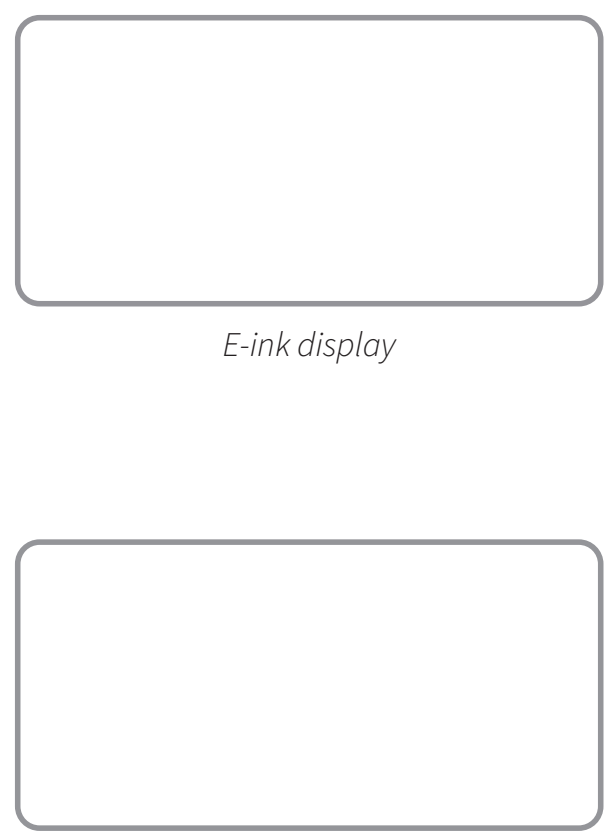

E-ink display

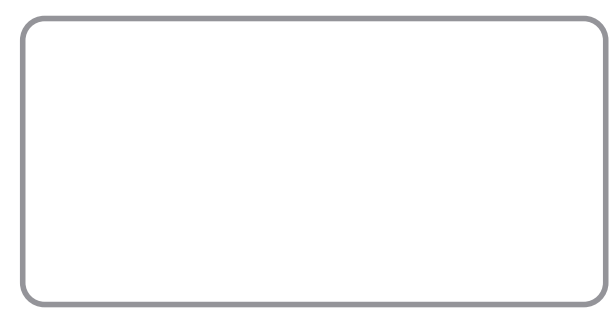

Esther needs to drink at least 20

oz of water today, but has only

consumed $6.5 \mathrm{oz}$ so far.

\section{Sleep mode}

The Bot is waiting for caregivers in sleep mode. It keeps sensing and monitoring data.

\section{Remind}

The Bot lights up to prompt caregivers to complete care activities that haven't been done on time.

\section{Turning the Bot}

In order to see details of the task, users need to turn the Bot so the right side glows up.

\section{Details of the task}

An e-ink display shows the detail of the task. 

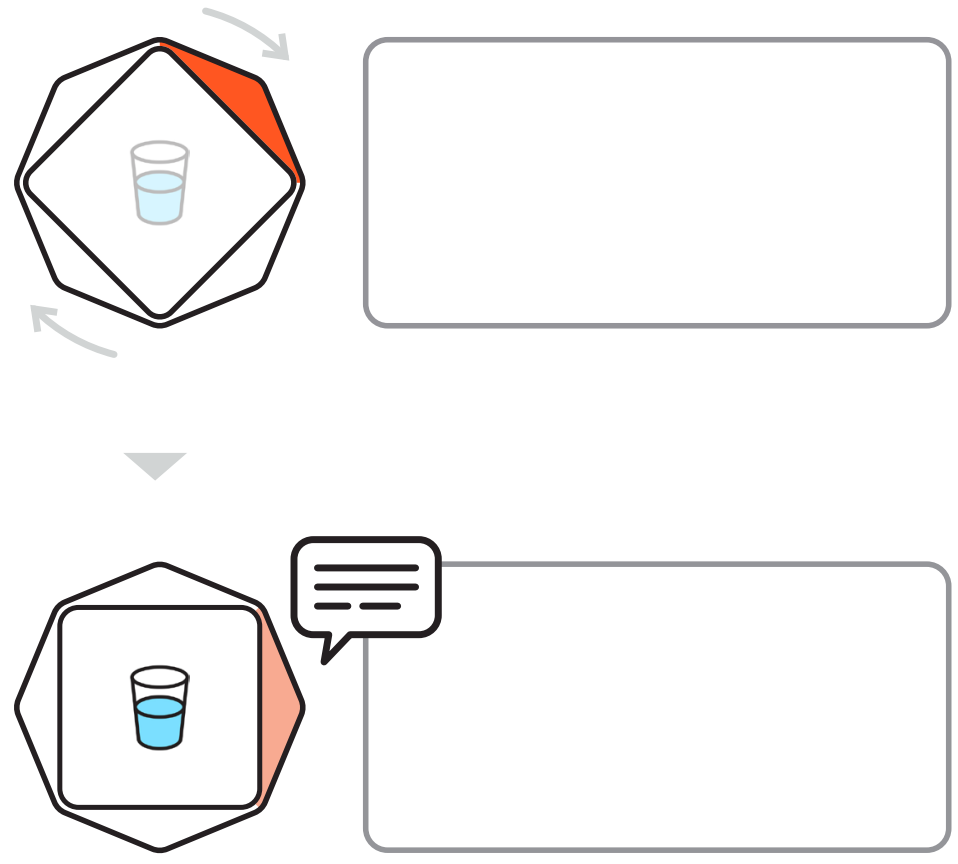

\section{Turning the Bot}

There is one more glowing side which indicates that it has an additional information to display. Users turn the Bot so the right side lights up again to see details.

\section{Details of the task}

The previous caregiver added a voice note to give more contextual information.

"I left a bottle for Esther in a fridge. Please make sure she finishes this by this even."

\section{When users want to add a voice note to keep other caregivers informed of specific contexts of care}

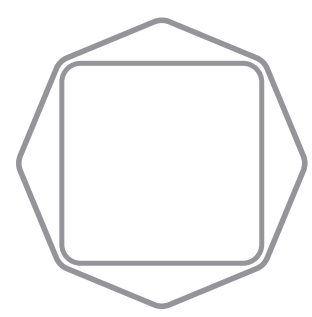

Bot

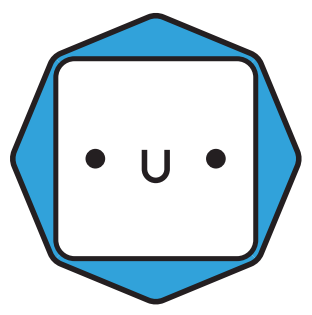

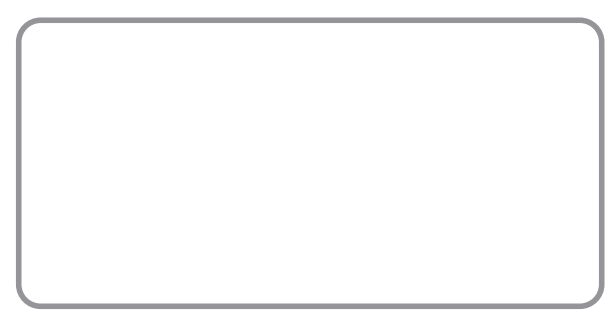

E-ink display

\section{Sleep mode}

The Bot is waiting for caregivers in sleep

mode. It keeps sensing and monitoring data.

\section{Motion detector}

The Bot spots users when they are in the room. When there is no reminder, the Bot glows in blue. 

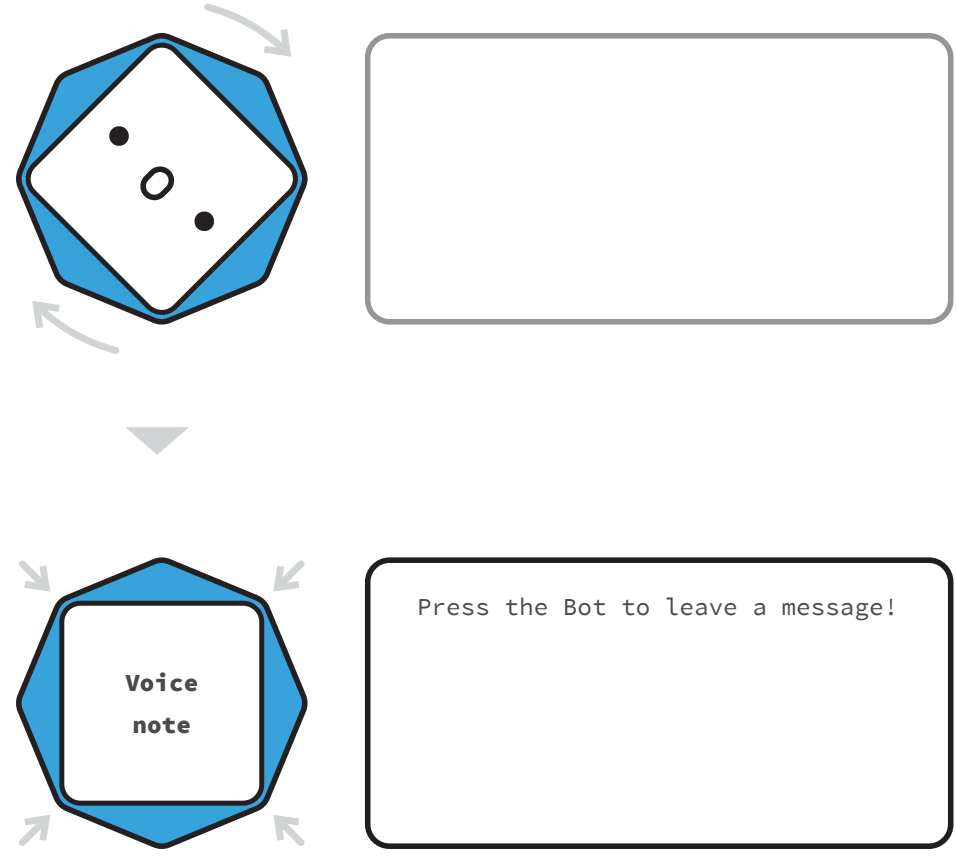

Press the Bot to leave a message!
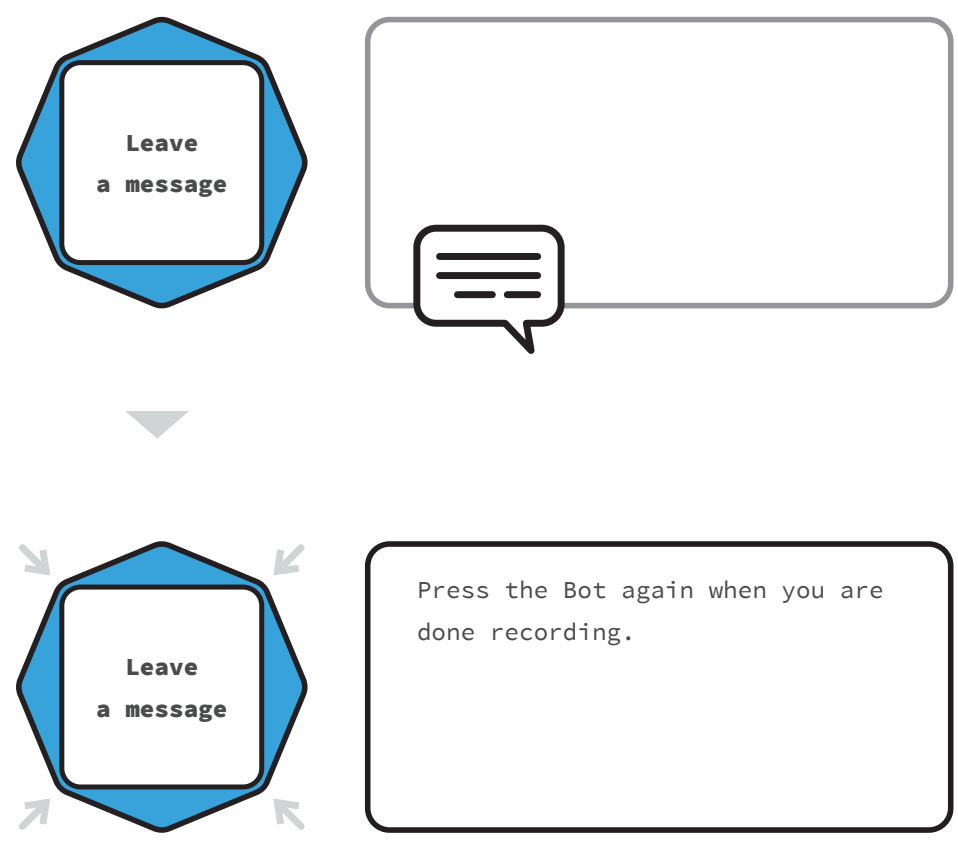

Press the Bot again when you are done recording.

\section{Turning the Bot}

In order to add a voice note, users need to turn the Bot.

\section{Pressing the Bot}

Then, press the Bot to start recording.

\section{Recording}

"I tried to help her stay active but she just wasn't in the mood."

\section{Finishing}

In order to finish recording, users need to press the Bot, and press the Bot again to confirm. 


\section{With the mobile application}

CareBot has a mobile application to keep all family caregivers in touch, informed, connected, and in control from anywhere. As described in Scenarios section, CareBot app is being used to filling in details of care information, such as profiles of family caregivers, regular schedule for must-do activities, medications, contact information and etc. Family caregivers who live away from the care recipient can be informed of and connected to care by getting a notification and participating in conversations through the app.

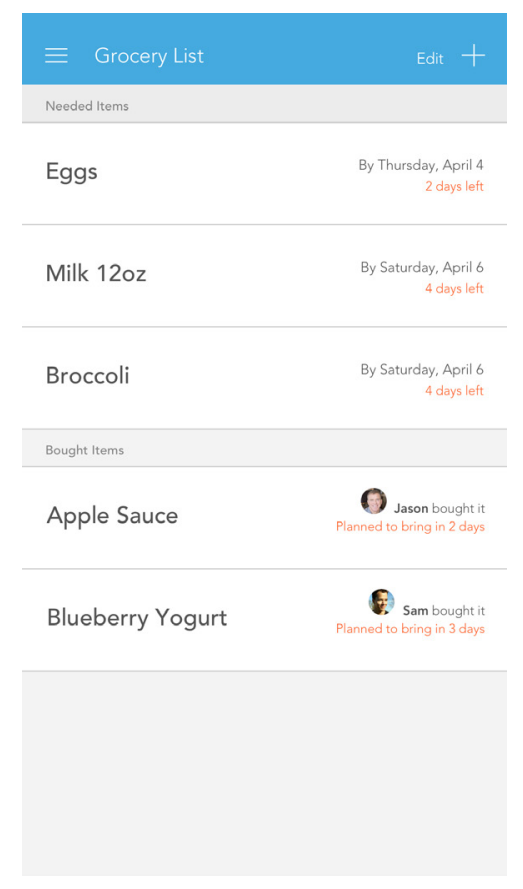
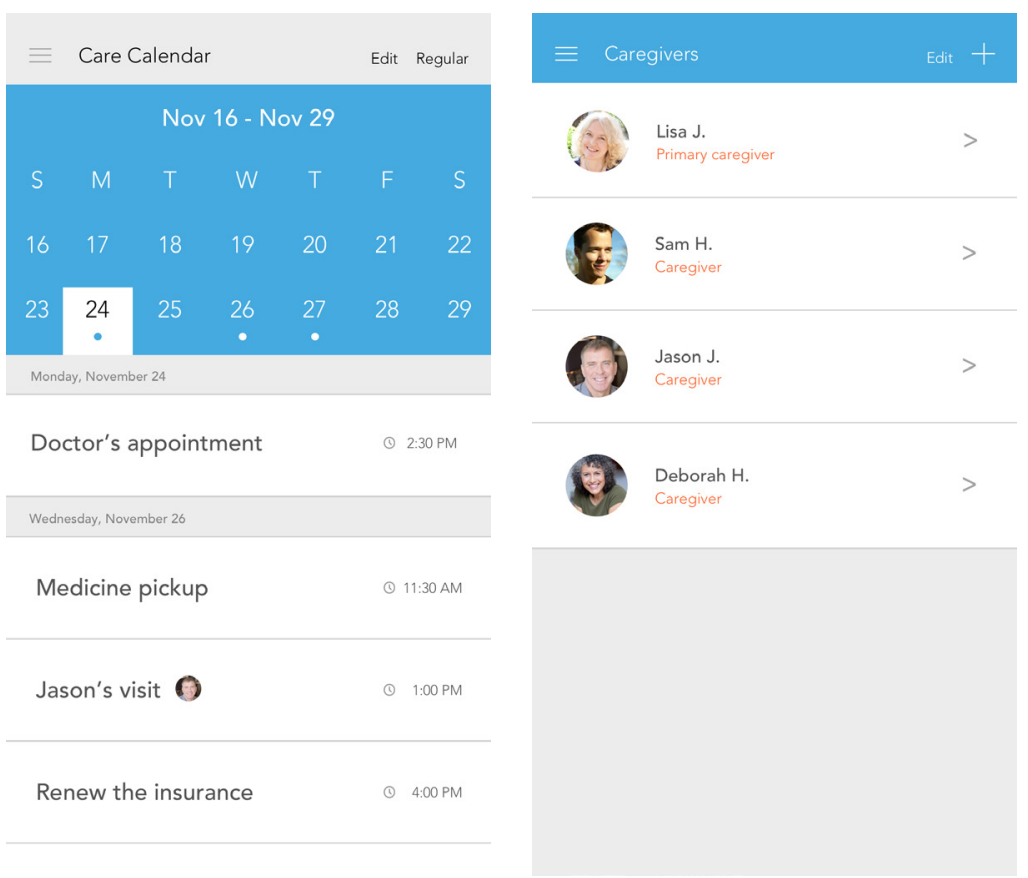

Fig. 23 Selected mobile screens - Grocery list, Care calendar, Caregivers profile 


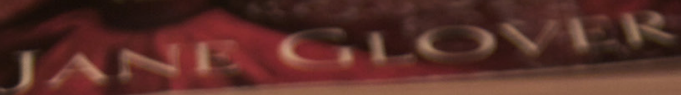

Fig. 26 The Bot welcomes

caregivers by lighting up the

smiley face when it detects

caregivers in the room.
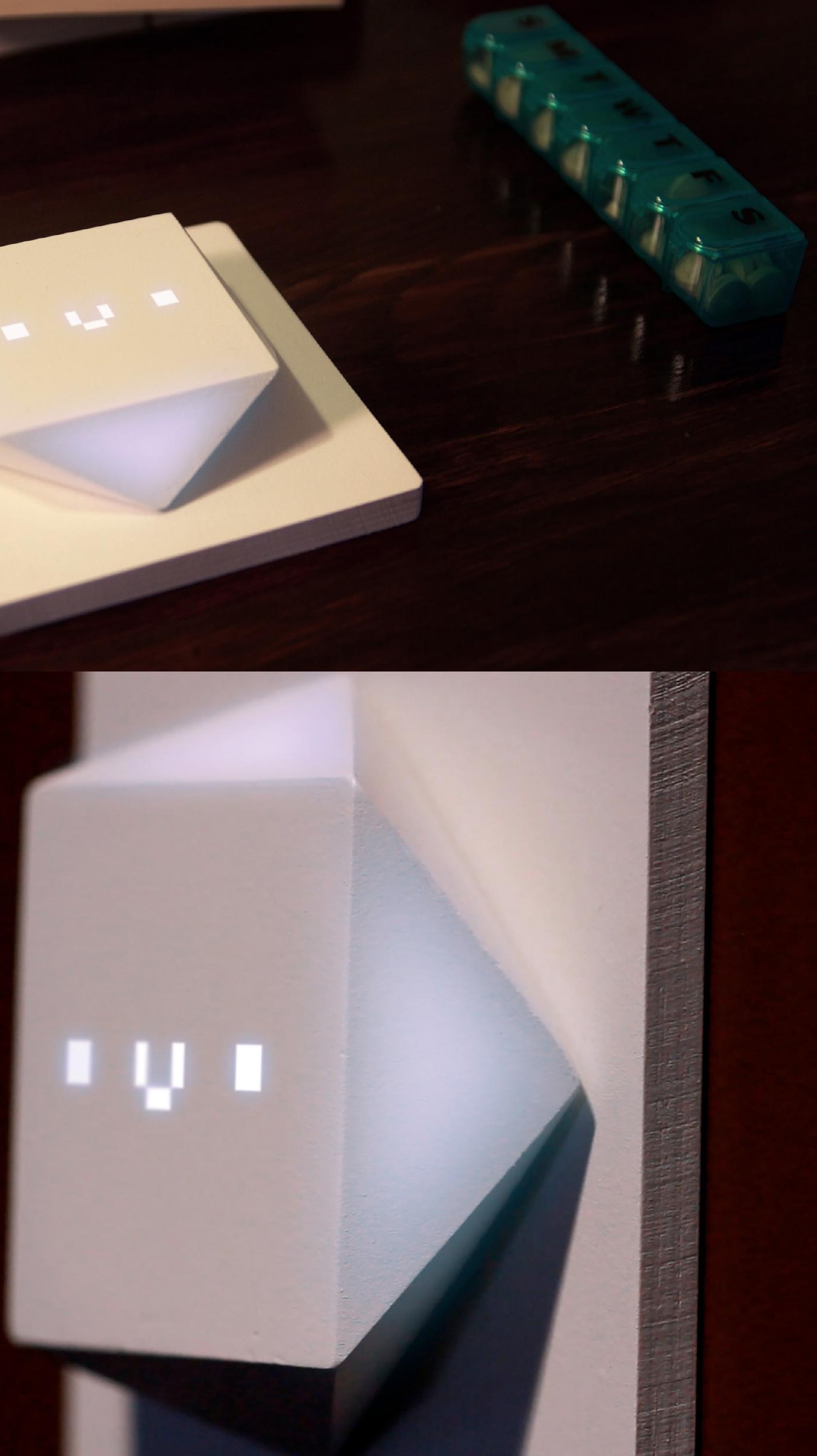


\section{Discussion}

\subsection{Social Dynamics}

Caregivers play a diverse range of roles in patient's care during a hospital stay. ${ }^{1}$ Likewise, caregivers who take care of care recipients in their own homes play many different roles in their care coordination according to their different territories. Different territories within an informal care network have different levels of obligation and interaction with a care recipient and other caregivers. In addition, these dynamics vary with different circumstances. Therefore, it is very important to create systems that have the fluidity in support of the dynamics of responsibility and social interaction of caregivers in different territories.

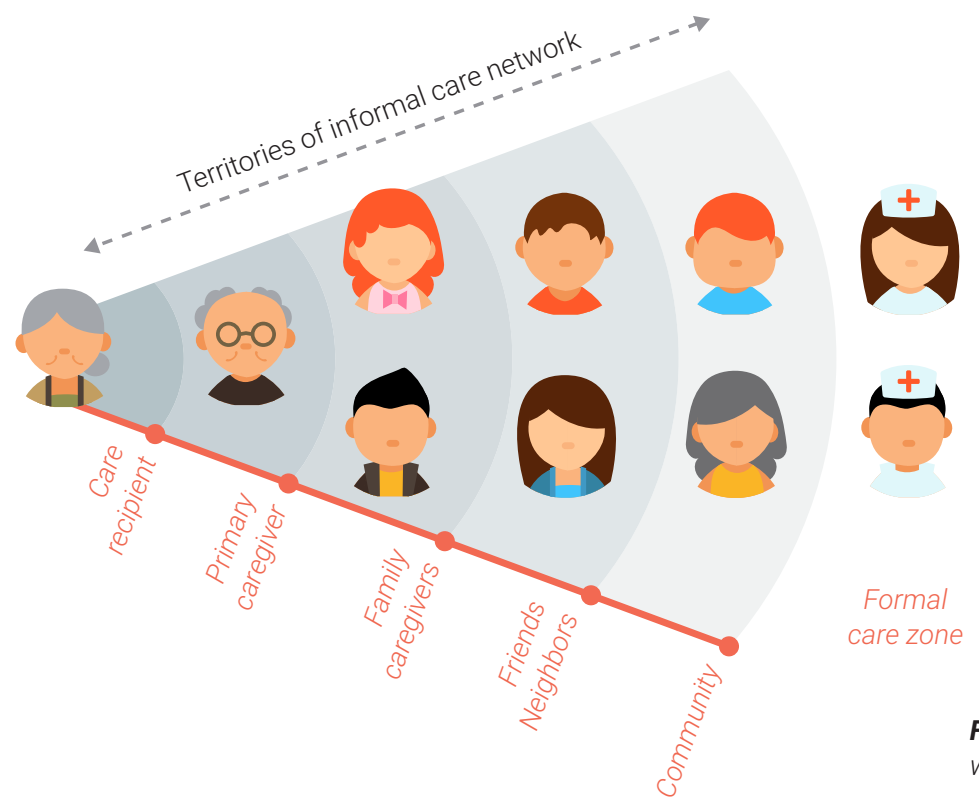

Fig. 27 Diverse territories within a care network

\subsection{Scalability}

As I move into the needs validation phase, I've discovered opportunities for the resulting design to benefit other kinds of coordinated activities in a home that are not only about a sick elderly person. The final concept can scale to a smart home platform for in-home coordination for a wide range of contexts, from feeding the dog

1 Andrew D. Miller, Sonali R. Mishra, Logan Kendall, Shefali Haldar, Ari H. Pollack, Wanda Pratt. Partners in Care: Design Considerations for Caregivers and Patients During a Hospital Stay, Proceedings of CSCW '16, San Francisco, CA, February 27-March 2, 2015. 
and watering the plant, to dual-income families' parenting.

\subsection{Limitations and Future Work}

As discussed earlier, the caregiver role involves a wide variety of types of care, and caregivers and care recipients' social dynamics are incredibly diverse. Although I tried to establish a spectrum of contexts of care, my research is still limited to certain types of contexts of care. For example, a majority of family caregivers that I met and spoke with were taking care of their care recipients who suffered from cognitive disabilities, such as dementia and Alzheimer's disease, and they are not necessarily representative of all informal caregivers. Therefore, further research should be conducted to evaluate against different disease and circumstances.

The concept of CareBot will also require a more robust system around it to better support family caregivers and care recipients. In the future, it can connect with a more diverse set of smart devices, such as a home medical kit, fitness kit, thermostat, and etc. The service side should be designed and verified as well in a holistic way in order to make the concept of CareBot more useful for family caregivers as a smart home platform. For example, family caregivers might want to seamlessly connect the information and data of their care activities that they gathered from CareBot to professional medical service providers for a better experience of medical care. Great opportunities exist for the bridge between informal care coordination and formal medical care, so further work is needed to explore these opportunities.

\section{Conclusion}

In this paper, I have presented details of the design process to explore opportunities for loT technologies to help informal caregivers better coordinate and communicate care with each other for their loved ones. The findings of the qualitative study on informal caregivers and their activities have revealed three main opportunities in which IoT can play a significant role for family caregivers' collective care coordination:

1. facilitating care works by reminding family caregivers of tasks that need to be done and providing instructions on how to accomplish it,

2. having crucial information accessible to everyone in the network of informal caregivers to stay organized and coordinated, and 
3. keeping everyone engaged and connected to avoid breakdown caused by poor communication and lack of information.

Based on these three main opportunities, I proposed the concept of CareBot, a smart home platform consisting of interactive tools in support of collective care activities of family caregivers. I believe the concept of CareBot can help family caregivers better coordinate and communicate care with each other and avoid potential breakdowns caused by having multiple caregivers who are cooperating to care for the same care recipient. 


\section{References}

"Aging in Place." Center for Aging in Place Support. Sep 2010

"An Aging Nation: The Older Population in the United States." U.S. Census Bureau, 2012 Population Estimates and 2012 National Projections. May 2014

Andrew D. Miller, Sonali R. Mishra, Logan Kendall, Shefali Haldar, Ari H. Pollack, Wanda Pratt. Partners in Care: Design Considerations for Caregivers and Patients During a Hospital Stay, Proceedings of CSCW '16, San Francisco, CA, February 27-March 2, 2015.

Arnall, T. (2014). Exploring 'Immaterials': Mediating Design's Invisible Materials. In International Journal of Design, Vol 8, No 2.

"Caregiving in the U.S. 2015", The National Alliance for Caregiving in collaboration with AARP

Baskinger, M., \& Gross, M. (2010). Tangible Interaction=Form+Computing. Interactions, 17 (1), ACM.

Bill Buxton. Chapter 13. Sketching Interaction. In Sketching User Experiences: Getting the Design Right and the Right Design. London: Morgan Kaufmann; 2010:136-140.

Buchenau, M. and Suri, J. F. (2000). Experience Prototyping. In Proceedings of Designing Interactive Systems. ACM Press: 424-433.

CareZone: http://carezone.com/home

Davidoff, S., Lee, M. K., Dey, A. K. and Zimmerman, J. Rapidly Exploring Application Design through Speed Dating. In Proc. of UbiComp, (2007) Springer, 429-446.

Dourish, P. (2001). Where the action is: the foundations of embodied interaction. The MIT Press.

Elizabeth Kaziunas, Ayse G Buyuktur, Jasmine Jones, Sung W Choi, David A Hanauer, and Mark S Ackerman. 2015. Transition and Reflection in the Use of Health Information. CSCW '15: Proceedings of the 18th ACM Conference on Computer Supported Cooperative Work \& Social Computing: 1763-1774. 
Frisardi, V.; Imbimbo, B.P. Gerontechnology for demented patients: Smart homes for smart aging. J. Alzheimers Dis. 2011, 23, 143-146.

Giovannetti ER, Wolff J, Xue Q, et al. Difficulty with health care tasks among caregivers of multimorbid older adults. J Gen Intern Med. 2012;27:37-44.

Hyewon Suh, John R Porter, Alexis Hiniker, and Julie A Kientz. 2014. @BabySteps: design and evaluation of a newstem for using twitter for tracking children's developmental milestones. CHI '14: Proceedings of the SIGCHI Conference on Human Factors in Computing Systems: 2279-2288.

Honor: http://joinhonor.com

Lê Q, Nguyen HB, Barnett T. Smart Homes for Older People: Positive Aging in a Digital World. Future Internet. 2012; 4(2):607-617.

Lee, M. K., Davidoff, S., Zimmerman, J. and Dey, A. K. Smart Bag: Managing Home and Raising Children. In Proc. of DPPI, (2007) ACM Press, 434-437.

Lee, M. L., \& Dey, A. K. (2010). Embedded assessment of aging adults: A concept validation with stakeholders. Paper presented at the 4th International Conference on Pervasive Computing Technologies for Healthcare (PervasiveHealth), 2010. Munich, Germany.

Liu LS, Hirano SH, Tentori M, Cheng KG, George S, Park SY, Hayes GR: Improving communication and social support for caregivers of high-risk infants through mobile technologies. In Proceedings of the Computer Supported Cooperative Work (CSCW) Conference. Hangzhou, China; 2011:475-484. New York, NY: ACM, USA; 2011.

Lotsa Helping Hands: http://lotsahelpinghands.com

Moncur, W., Masthoff, J., Reiter, E., Freer, Y., \& Nguyen, H. (2014). Providing Adaptive Health Updates across the Personal Social Network. Human-Computer Interaction, 29(3), 256-309.

Morris, Meg E., Adair, Brooke, Miller, Kimberly, Ozanne, Elizabeth, Hansen, Ralph, Pearce, Alan J., Santamaria, Nick, Viega, Luan, Long, Maureen and Said, Catherine M. 2013, Smart-home technologies to assist older people to live well at home, Journal of aging science, vol. 1, no. 1, pp. 1-9. 
"The New Era of Connected Aging: A Framework for Understanding Technologies that Support Older Adults in Aging in Place." Center for Technology and Aging Report. 2014

Yunan Chen, Victor Ngo, and Sun Young Park. (2013). Caring for caregivers: designing for integrality. CSCW '13: Proceedings of the 2013 conference on Computer supported cooperative work: 91-102. 
